\title{
The effects on priming of word frequency, number of repetitions, and delay depend on the magnitude of priming
}

\author{
ARNE L. OSTERGAARD \\ University of Califormia, San Diego, La Jolla, California
}

\begin{abstract}
Conflicting findings with respect to the effects of experimental manipulations on priming have been reported in previous studies. It is argued that, in many priming tasks, large amounts of task-relevant information are available from various sources, and that, therefore, the information available from a specific study episode will have only a small impact on overall performance level. Under such circumstances, high levels of baseline performance and small priming effects will be observed. The experiments reported here investigated the hypothesis that a high baseline performance in informationprocessing tasks that are used to measure priming may constrain priming effects. In a series of wordnaming experiments, perceptual difficulty and, therefore, baseline performance was manipulated. Under easy conditions, priming effects were relatively small and were not affected by word frequency, spaced repetition, or delay. Under more difficult conditions, priming effects were larger, and significant effects of all of the above-mentioned experimental manipulations were observed. Under conditions that produced the largest priming effects, a significant relationship between priming and explicit-memory performance could be observed. In the last experiment, it was shown that the characteristics of the retrieval task can substantially affect the magnitude of priming. It is argued that priming effects should be considered to reflect interactions between memory traces and the information-processing components of the priming task.
\end{abstract}

Experimental manipulations that significantly affect explicit-memory performance on tasks such as recall or recognition often have little or no significant effect on priming measures that are obtained in tasks such as tachistoscopic identification, lexical decision, and word-stem completion. These variables include massed repetition of studied items, divided versus focused attention, and levels of processing of the study items (Greene, 1990; Jacoby \& Dallas, 1981; Parkin, Reid, \& Russo, 1990). Such functional dissociations between memory measures have led to the suggestion that independent implicit- and explicitmemory systems exist (Squire, 1992; Tulving \& Schacter, 1990). However, the effects of experimental variables on priming are often inconsistent from priming task to priming task, and even when the same task is used, they may not be consistent from study to study.

The present investigation is concerned with the effects of three such variables on priming — word frequency, spaced

This work was supported by Grant AGO6849 from the National Institute on Aging and by Grant AA09465 from the National Institute on Alcohol Abuse and Alcoholism. For their assistance in collecting the data, I thank Catalina Amaya, Lauren Norton, and Julie Sellers. I also thank Michael Masson, Bruce Whittlesea, and Alan RichardsonKlavehn for their comments on an earlier draft. Correspondence concerning this paper should be addressed to A. L. Ostergaard, University of California, San Diego, Human Memory Laboratory 0949, 9500 Gilman Drive, La Jolla, CA 92093 (e-mail: aostergaard@ ucsd.edu).

-Accepted by previous editor, Geoffrey R. Loftus repetition, and delay between study and test. It is noted, however, that other variables, such as levels of processing and divided versus focused attention, also have produced conflicting results with respect to their effects on priming measures (see, e.g., Eich, 1984; Parkin et al., 1990; Squire, Shimamura, \& Graf, 1987).

A common finding in studies of perceptual priming is that low-frequency words are associated with larger priming effects than are high-frequency words. Such frequency effects on priming have been obtained in lexicaldecision tasks, perceptual-identification tasks, and wordfragment completion tasks (Jacoby \& Dallas, 1981; Kirsner, Milech, \& Stumpfel, 1986; MacLeod, 1989; Scarborough, Cortese, \& Scarborough, 1977). However, these effects of frequency on priming often are small and not statistically significant. For instance, in two lexical-decision experiments reported by Scarborough, Gerard, and Cortese (1979) and in one out of three lexical-decision experiments reported by Scarborough et al. (1977), the effect of word frequency on priming failed to reach significance. Hintzman and Hartry (1990) found virtually no correlation between the magnitude of priming and rated familiarity (presumably closely related to frequency) in wordfragment completion. Finally, Scarborough et al. (1977) found no hint of a word-frequency effect on priming in a pronunciation (naming) task.

With respect to repetition effects on priming (i.e., the number of times primed items have been studied prior to the priming test), existing findings are also conflicting. A distinction is made between massed and spaced repe- 
tition. In massed repetition, a stimulus is repeated on successive trials without any other items intervening between the repetitions. In spaced repetition, one or several items intervene between the repeated presentations of a stimulus. In general, massed repetition does not significantly increase the magnitude of priming over that observed with a single presentation of the primed items (Challis \& Sidhu, 1993; Greene, 1990; Jacoby \& Dallas, 1981; Perruchet, 1989; Roediger \& Challis, 1992). When repetitions are spaced, the evidence is more confusing. Jacoby and Dallas (1981) found a significant effect of spaced repetition on priming in one tachistoscopic wordidentification experiment, but not in another. Feustel, Shiffrin, and Salasoo (1983) reported effects of spaced repetitions in a word-identification task using a perceptual-clarification procedure, whereas Perruchet (1989), using a similar procedure, obtained a significant effect of spaced repetition in only one of four experiments. Greene (1990) found effects of spaced repetitions on priming in homophone-biasing and word-fragment completion tasks. However, in tachistoscopic word identification, Greene obtained an effect of spaced repetition on priming under intentional, but not incidental, learning conditions.

Early priming studies seemed to indicate that priming effects could be remarkably persistent and show little or no decline over periods of days or even weeks (Jacoby \& Dallas, 1981; Tulving, Schacter, \& Stark, 1982). Subsequently, it has become clear that, given long enough retention intervals, priming effects eventually do decline significantly (see Richardson-Klavehn \& Bjork, 1988; Roediger \& McDermott, 1993). However, studies using comparable retention intervals have produced conflicting results. Some investigators have reported that priming in word-stem completion declines to chance levels within about $2 \mathbf{h}$ when the stems have many possible solutions, whereas priming in word-fragment completion shows little or no decline over this period (Graf \& Mandler, 1984; Graf, Squire, \& Mandler, 1984; Squire et al., 1987). Roediger, Weldon, Stadler, and Riegler (1992) suggested that such differences between word-stem and word-fragment completion tasks could be due to the fact that longer and less common words are used in wordfragment completion tasks than are used in word-stem completion tasks. Consequently, Roediger et al. (1992) employed the same materials in word-stem and wordfragment completion tasks and found that, under those conditions, priming in the two tasks showed similar patterns of decline over time. There was little or no decline of priming in either word-stem or word-fragment completion over delays of up to $2 \mathrm{~h}$, but after longer (48- to 168-h) delays, a decline was observed. Jacoby and Dallas (1981) failed to find a significant decline of priming in tachistoscopic word identification over a 24-h interval, whereas Jacoby (1983a) did report a significant decline of priming after the same interval.

It is clear that the effects of many experimental manipulations on priming are variable and perhaps taskdependent. Ostergaard (1994) obtained similarly conflict- ing evidence in a direct comparison between two perceptual word-priming tasks. Delay of the priming test and the number of repetitions of the primed items affected the magnitude of priming in a tachistoscopic word-identification task, but not in a task of identifying visually fragmented words. Ostergaard (1994) illustrated how the characteristics of the retrieval cues provided in the priming task could produce such divergent results. Different information-processing tasks may not be equally sensitive to, or facilitated by, a given level of encoded information. Furthermore, the magnitude of facilitation in an informationprocessing task may not be linearly related to the strength of encoded and stored information. Such nonlinearities between different priming measures - and between any given priming measure and memory-trace strength (possibly because of the influence of floor or ceiling effects) could produce both apparent dissociations between measures and conflicting results with the same tasks (Dunn \& Kirsner, 1988; Neely, 1989; Olton, 1989). It seems clear that priming effects should be considered within a broader context of general information processing. It may be fruitful to distinguish between encoding, storage, and retrieval processes in priming as well as in explicitmemory tasks. This is the line of reasoning that forms the basis for the present experiments. In particular, priming effects are considered to be the results of interactions between retained information and the informationprocessing components of the priming tasks. In other words, priming effects are not considered to be direct reflections of underlying memory representations.

A corollary of this view is that an understanding of priming effects requires knowledge of the specific processing components of the task - that is, of the baseline information-processing task. Unfortunately, in many cases such tasks are poorly understood, and the information-processing requirements of priming tasks are often ignored in priming studies. However, if a manipulation changes baseline performance level in a task, then some aspects of the information processing required to carry out the task must have changed. The experiments reported here investigate the consequences for priming effects of the manipulation of baseline performance in a word-naming task.

\section{Theoretical Perspective: Information Availability}

In priming tasks, the subject is often required to identify or make decisions about presented stimuli. Subjects may be required to identify and name stimuli that are presented in a complete form (e.g., words), very briefly in a tachistoscope, or in some incomplete form (e.g., visually degraded words or word stems). Two examples of decisions that subjects may be required to make in priming experiments are deciding whether a presented letter string is a real English word (lexical decision) or deciding whether a picture is depicting an animate or an inanimate object (semantic classification). It seems reasonable to assume that the subject will use any relevant information from any source to carry out such tasks. The discussion that fol- 
lows will focus on tasks in which the subject attempts to identify visual stimuli, but the arguments apply to other tasks and modalities.

In visual-identification tasks, the information available to the subjects may be regarded as coming from three sources. First, there is the perceptual information that is directly available from the stimulus $(P)$. The amount of stimulus information available depends on how the item is presented. More $P$ will be available from a stimulus that is complete and clear than from one that is degraded or presented very briefly. Stimuli that are presented as word stems or word fragments contain less $P$ than items that are complete words. In order to achieve successful identification, the $P$ has to be integrated with information retrieved from memory. The information in memory that is relevant to the identification of the item is the information that has been accumulated and is still available from all prior encounters with the item. With respect to priming tasks (and indeed other memory tasks), this information in memory may be regarded as coming from two sources. There is the information that is available in memory from a specific priming or study episode $(S)$, and there is the information that is available from all other prior encounters with the item $(O)$. The information from a prior study or priming episode is, of course, available only for previously studied items and not for items used to determine the baseline performance level. This study episode can often be assumed to represent the subject's most recent encounter with the primed items. However, this is not always the case. If the study episode involves relatively common words, and if the priming test is carried out after a relatively long retention intervalsay, a week (see, e.g., Tulving et al., 1982)-it seems likely that subjects will have encountered many of the study words during the retention interval. The information that is available from all other prior encounters with an item will vary from item to item. More information will be available for familiar or frequently occurring words than for rare or unfamiliar items. No distinction is made between semantic, lexical, or structural information. Any information that has accumulated in memory from prior encounters with an item that is relevant to the task at hand falls into this category.

Thus, the information that is available to a subject in an identification task is the perceptual information directly available from the stimulus $(P)$, the information that remains in memory from a specific prior study episode ( $S$, available only for previously studied items), and the information that has accumulated in memory from all other prior encounters with the item $(O)$. A central tenet of the argument made here is that the subject will use any relevant information from these three sources to accomplish the task. Consequently, the extent to which information from any one of these sources will affect performance in a task depends not only on how much information is available from that specific source, but also, to a large extent, on how much information is available from the other sources. Specifically, for familiar items that are presented clearly, large amounts of $P$ and $O$ may be available relative to the $S$ available from a prior study episode; therefore, the prior study episode may have only a small impact on overall performance (the priming effect will be small and perhaps not detectable).

When a subject is attempting to identify an item, the subject retrieves relevant information from memory and integrates it with the stimulus information. In identification tasks, the stimulus cue determines what information in memory is relevant. The subject searches for and retrieves information that is consistent with the cue; therefore, what is retrieved is, in some sense, constrained by the information provided by the stimulus $(P)$. This process may be conceived of as consisting of a series of search-decision cycles. The subject searches memory for relevant information, and, at regular intervals, a decision is made as to whether the item can be identified. If the item can be identified, a response is made. If it cannot yet be identified, another search-decision cycle is carried out. Therefore, if it takes $n$ search cycles to accomplish a complete search of memory, then, on average, $1 / n$ of the relevant information in memory will be retrieved in each cycle. That is, $S / n$ of the information from the study episode (if any) and $O / n$ of the information from all other encounters with the item will be retrieved during each cycle. Assuming that a certain threshold $(T)$ amount of information has to be exceeded in order to identify the item, and given that $P$ is the amount of information available directly from the stimulus, then the amount of information that has to be retrieved from memory is $T-P$. Since the amount of relevant information retrieved from memory in each search-decision cycle is $O / n+$ $S / n$, the number of cycles $(j)$ required to identify an item is given by

$$
j \geq \frac{T-P}{\left(\frac{O}{n}+\frac{S}{n}\right)},
$$

which implies

$$
j \geq \frac{n \cdot(T-P)}{(O+S)} .
$$

The number of search-decision cycles $(j)$ required to identify the item is the smallest integer satisfying Equation 1 . Response time will, of course, be directly related to $j$. The important aspect of such a system is that all three sources of information interact. The effect of variation in familiarity $(O)$ with stimulus items depends on the amount of perceptual and study information available, and the effect of prior study depends on the amount of perceptual and other information available.

Figure 1 illustrates this point with respect to the effect of prior study (priming) on identification performance. In that illustrative example, it is assumed that 100 arbitrary units of information are required for identification $(T)$ and that 50 units of perceptual information are directly 
A: Effects of Varying Stimulus Familiar ity $(O)$

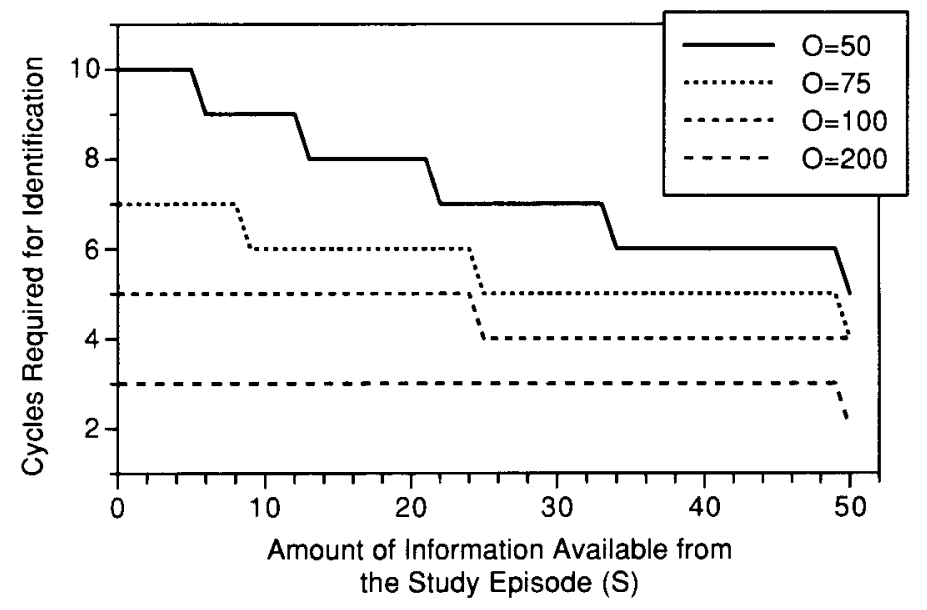

B: Effects of Varying Stimulus Degradation (P)

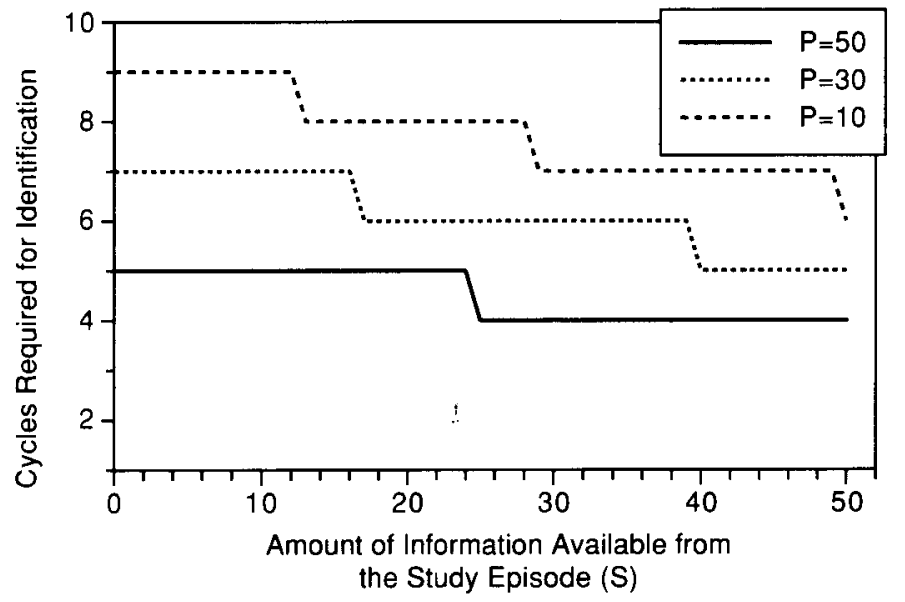

Figure 1. The consequences for identification performance, as predicted by the in formation availability model, of varying stimulus familiarity $(O)$, stimulus degradation $(P)$, and the amount of information available from a prior study episode $(S)$. It is assumed that 10 search-decision cycles are required to accomplish a complete memory search, and that 100 arbitrary units of information are required to identify an item $(T)$. Both panels show identification performance in terms of search-decision cycles as a function of the amount of information available from a prior study episode $(S)$. A value of $S=0$ represents baseline performance level (i.e., the item did not occur in the study episode). Panel $A$ shows the consequences of varying item familiarity - that is, the information available from preexperimental encounters with an item $(O)$ - for items presented clearly ( 50 units of perceptual information $[P]$ are available directly from the presented stimulus). Panel $B$ shows the consequences of degrading a moderately familiar item $(O=100)$, such that less perceptual information $(P)$ is directly available from the presented stimuli.

available from the stimulus $(P)$. It is further assumed that it takes 10 search-decision cycles to accomplish a complete memory search. The horizontal axis represents the information (0-50) available in memory from a specific prior study episode. A value of zero on the horizontal axis, of course, represents a condition in which there was no prior study episode- that is, a baseline condition. The vertical axis represents the number of search-decision cycles required to identify an item. The top panel (A) i1lustrates the consequences of variations in familiarity (i.e., the availability in memory of information $(O)$ from all other prior encounters with the item) on measured priming effects. First, familiarity (amount of $O$ available) affects baseline identification performance; the less familiar items $(O=50, O=75)$ require considerably more search-decision cycles for baseline identification 
than do the more familiar items $(O=100, O=200)$. The important point, however, is that the familiar items can produce only very small priming effects and are not capable of revealing variations in priming, regardless of how much information is available in memory from a prior study episode. The very familiar item can be identified at the third search-decision cycle, regardless of whether 0 or 45 units of information are available in memory from a prior study episode. Such an item can show priming only if very high levels of information are available from a prior study episode; generally there will be very little relation between the amount of information available in memory from the study episode and the size of obtained priming effects. The moderately familiar item $(O=100)$ will produce a priming effect when 25 units of prior study information are available in memory. However, the magnitude of priming obtained with such an item will remain unchanged for any variation in the amount of information available from a prior study episode between 25 and 50 units. In other words, the amount of information available can be doubled without having any observable effect on measured priming. Again, there will be only a very weak relationship between the amount of information that is available in memory from a specific prior study episode and measured priming effects.

The bottom panel of Figure 1 illustrates the consequences of manipulating the availability of perceptual information for a moderately familiar item $(O=100)$. As the stimulus is degraded from $P=50$ through $P=30$ to $P=10$, baseline identification $(S=0)$, of course, becomes slower and slower. However, the important point is that priming effects become larger and more sensitive to variations in the amount of information available from a prior study episode as the stimulus is degraded and less and less perceptual information is available.

Strictly speaking, Equation 1 is only correct if the perceptual information from the stimulus becomes available very rapidly in comparison with the time it takes to retrieve information from memory. Specifically, the equation is valid only in cases in which all perceptual information has been extracted from the stimulus by the time at which the item can be identified. This is unlikely to be true in all cases. The present investigation employs a form of visual degradation whereby the stimulus is gradually clarified over a short period of time. Similar procedures have been used in previous studies (e.g., Johnston, Dark, \& Jacoby, 1985; Perruchet \& Baveux, 1989). Under such circumstances, the rate at which perceptual information becomes available is manipulated, and this rate, rather than the total information in the complete stimulus, has to be specified. Thus if $p$ is the amount of perceptual information $(P)$ that becomes available during each searchdecision cycle, the total amount of information that becomes available during a cycle is

$$
\frac{O}{n}+\frac{S}{n}+p
$$

Since $T$ is the total amount of information required for identification, the number of cycles required for identification $(j)$ is given by:

$$
j \geq n \cdot \frac{T}{O+S+n \cdot p} .
$$

Equation 2 is the equivalent of Equation 1 for tasks in which the stimulus is not presented at a fixed level of degradation but is gradually clarified. Manipulating the rate at which perceptual information becomes available $(p)$ will, of course, produce effects that are similar to those caused by manipulating the other variables in the denominator of Equation 2. Specifically, reducing the rate at which perceptual information becomes available will increase baseline naming latency, increase the effects of variability of stimulus familiarity $(O)$, and increase the magnitude and variability of measured priming effects.

The exact values used in these examples are not important; it is the relationship between the availability of information from various sources that matters. Familiar items presented clearly may produce relatively small but consistent priming effects, although the magnitude of these effects may not be strongly related to the amount of information that is available from a prior study episode. Therefore, experimental manipulations that affect the amount of information available from a prior study episode (e.g., the amount of time for study or the length of delay between study and test) may have no, or only little and perhaps undetectable, effects on measured priming. In other words, priming measures may be insensitive to even relatively large variations in the amount of study information available in memory.

An important aspect of the present model is that, given that the amount of study information is kept constant, the factors that affect priming effects also affect baseline identification performance. Baseline performance is determined jointly by the amount of information required for the subjects to complete the task $(T)$, the availability of perceptual information $(P)$, and the availability in memory of information from prior encounters with a stimulus $(O)$. For a given amount of information available from a specific study episode, the magnitude of measured priming effects is also determined by these same factors ( $T, P$, and $O$ ). Therefore, the model predicts a strong relationship between baseline performance level and measured priming effects. It should be possible to increase measured priming effects in a task by using unfamiliar rather than familiar items or by degrading the stimuli such that less perceptual information is available. Reduced levels of baseline performance should be associated with increased magnitude of priming effects. If baseline performance is regarded as an index of task difficulty, according to the present hypothesis, there is a direct relationship between task difficulty and measured priming. This relationship between baseline performance and measured priming is important when memory-impaired patients are compared with normal control subjects. If the patients 
have deficits that affect their baseline identification performance, then these have to be taken into account when evaluating the priming effects (see Hamann, Squire, \& Schacter, 1995; Jernigan \& Ostergaard, 1993; Ostergaard, 1994; Ostergaard \& Jernigan, 1993, 1995).

In summary, the magnitude and variability of priming effects that can be obtained in a given task may be constrained by the availability in memory of relevant informaticn other than that from the prior study episode. In effect, there may be a ceiling on the priming effects that can be observed. An item that can be identified at the first search-decision cycle in the baseline condition cannot, of course, show any priming, regardless of how much information is retained from a prior study episode. An item that can be identified at the second search-decision cycle can show a priming effect of only one cycle, and so on. Thus, priming effects may be regarded as being constrained by baseline performance levels.

In the present experiments, baseline word-naming performance is manipulated by reducing the amount of perceptual information that is available to the subject. It is expected that, as the availability of perceptual information is reduced (i.e., as task difficulty is increased), constraints on measured priming effects will be reduced and, therefore, the magnitude of these effects will increase. Furthermore, it is expected that the effects of experimental manipulations on priming will become detectable as the constraints imposed on priming effects by high levels of baseline performance are reduced.

\section{GENERAL METHOD}

The same word-naming task was employed in all four experiments reported in the present paper. This fade-in task was designed to allow control over baseline performance in word identification. In the task, items presented on a computer screen come gradually into view (fade in). The task is similar to the "perceptual clarification" procedures used by Johnston et al. (1985) and by Perruchet and Baveux (1989). Words can appear instantly, or they can fade in on the computer screen over a period of up to $5 \mathrm{sec}$.

The presentation of the words is controlled by an Apple IIGS microcomputer, and the fade-in appearance of items is achieved by gradually turning on more and more pixels of the presented items. The color palette is changed such that two pixel values (B1 and B2) both produce the background color (white in the present case) and a third pixel value $(\mathrm{F})$ generates the desired foreground color (black in the present case). Items are presented in a 5,120-pixel window (height $=16$, width $=320$ ). First, the window is cleared by changing all pixel values to $\mathrm{Bl}$. Then a warning signal, consisting of three plus signs $(+++)$, is drawn in the foreground color, and this remains on the screen for $1 \mathrm{sec}$ after which all pixel values are again set to $\mathrm{B} 1$ (the window is cleared). Then the target item is drawn using the second background pixel value (B2), which is invisible on the screen because these pixels are displayed in the same background color as the true background pixels $(\mathrm{B} 1)$. The fade-in algorithm is then implemented $0.5 \mathrm{sec}$ after the offset of the warning signal. Each of the 5,120 pixels is examined once, in a pseudorandom order, and if an examined pixel is of value $B 2$ (i.e., is part of the item), its value is changed to that of the foreground color $(F)$. Pixels of value $B 1$ (true background pixels), are, of course, not changed. The number of pixels examined each millisecond can be varied, so that a word can occur instantly (all pixels revealed immediately), or it can fade in over a period of up to $5.12 \mathrm{sec}$ (one pixel examined per millisecond). The start of the fade-in algorithm is synchronized with the vertical blanking interval (start of the screen retrace) and with the start of the response timer. Naming-response latencies are measured using a microphone connected to a voice-activated relay, which, when activated, produces a game-port input to the computer. Latencies are recorded in milliseconds by the computer.

With this procedure the items appear to fade in continuously and smoothly, and, since all 5,120 pixels in the window are always examined during a fade-in event, all words (short or long) fade in at the same predetermined rate.

Words were presented in capital letters and each letter was $9 \mathrm{~mm}$ high and $6 \mathrm{~mm}$ wide. The words employed in the experiments were drawn from a pool of 900 nouns, ranging in length from four to nine letters and in Kučera and Francis (1967) frequency counts from 1 to 291 .

\section{EXPERIMENT 1}

Word frequency is a factor that, in previous studies, has had variable effects on priming, as discussed above. Scarborough et al. (1977) found significant effects of word frequency on priming in two lexical-decision experiments, but failed to find any hint of a frequency effect on priming in a pronunciation (naming) task with similar materials. Two aspects of the results obtained by Scarborough et al. (1977) are important in the present context. Baseline response latencies were longer and priming effects were larger in the lexical-decision task than in the pronunciation task (see Scarborough et al., 1977, Figure 7). The fact that baseline response latencies were longer in the lexical-decision task than in the pronunciation task suggests that the lexical-decision task was more difficult than the pronunciation tasks. In other words, it was necessary to collect more information $(T)$ to carry out the lexical-decision task than to carry out the pronunciation task. Increasing the amount of information $(T)$ that is required to carry out a task, within the present framework, has the same effect as reducing the availability of perceptual information that is available from the stimulus-it will increase the magnitude and the sensitivity to experimental manipulations of priming effects. Thus, the results reported by Scarborough et al. (1977) are consistent with the idea that priming effects were more constrained by high levels of baseline performance in the pronunciation task than they were in the lexical-decision task.

Experiment 1 attempts to reproduce both of these findings in a naming (pronunciation) experiment. Baseline naming latency is manipulated in a word-naming task by presenting the words either instantly or gradually over a period of $1.7 \mathrm{sec}$. The instant condition represents a partial replication of Scarborough et al.'s (1977) Experiment 3; therefore, little or no effect of word frequency on priming is expected in that condition. However, the present hypothesis is that the lack of a frequency effect on priming in word naming is due, at least in part, to the presence of constraints on the obtainable priming effect because of high levels of baseline word-naming performance. The 
fade-in manipulation is expected to reduce these constraints by reducing the availability of perceptual information; consequently, word frequency is expected to affect priming when the items are presented gradually.

\section{Method}

Subjects. Twenty University of California, San Diego, students participated in this experiment. They were paid at a rate of $\$ 8$ per hour.

Design and Materials. A three-way within-subjects factorial design was employed. The first factor was the speed with which the stimuli were revealed-instantly or over a period of $1.7 \mathrm{sec}$. The second factor-word frequency --had three levels: low-frequency words (mean Kučera \& Francis, 1967, frequency count $=2.6$ ), mediumfrequency words (mean Kučera \& Francis, 1967, frequency count $=$ 16.7), and high-frequency words (mean Kučera \& Francis, 1967, frequency count $=152$ ). Because the words were repeated, the third factor was occurrence - that is, first or second occurrence.

Two blocks of trials were constructed, each consisting of 60 target words and 30 filler words. Within a block, each of the 60 target words occurred twice. The target words consisted of 20 low-, 20 medium-, and 20 high-frequency words. In each frequency category, there were 4 words with each of the following lengths: five letters, six letters, seven letters, eight letters, and nine letters. The order of the resulting 120 critical trials within a block was randomized, with the following restrictions: the lag between the two occurrences of target items was random within the range of 15 to 20 items, and repeated words occurred on no more than four successive trials. Twenty-five of the filler words were interspersed among the critical trials to satisfy these criteria. Additionally, the first 5 trials in each block were regarded as practice trials, and the words used in these trials were filler words. In short, in each block of 150 trials, 60 target words (20 low-, 20 medium-, 20 high-frequency) were presented twice, with lags varying from 15 to 20 items. One block was used in the slow fade-in condition and the other block in the instant condition. The two blocks were rotated between fade-in conditions across subjects.

Half the subjects were given the instant condition before the slow fade-in condition, and half the subjects were given the slow fade-in condition first.

Procedure. Stimulus presentation and the recording of response latencies were controlled by an Apple IIGS microcomputer, as described above.

The subject was seated at a comfortable viewing distance in front of the computer and was instructed that a long series of words would be presented on the computer screen, each preceded by a warning signal consisting of three plus signs $(+++)$. The subject was told to read aloud (name) each word as quickly as possible without making errors. Prior to each condition, the subject was informed whether the words would appear instantly or fade in gradually. The subject was also informed that many words would be repeated.

The presentation of warning signals and stimulus words was carried out as described above; each word remained on the screen for $3 \mathrm{sec}$. After the subject named a word, the examiner hit the return key if the response was correct and the spacebar if it was wrong. The subject was given a short rest (approximately $10 \mathrm{~min}$ ) between the two blocks of 150 trials.

\section{Results and Discussion}

In all statistical analyses reported below, the probability of Type I error of significant results is $p<.05$. In this and all subsequent experiments, response latencies were excluded from all analyses involving latencies according to the following criteria: latencies associated with incor- rect responses were excluded; latencies of $200 \mathrm{msec}$ or less were attributed to an accidental triggering of the voice switch and were excluded; latencies that deviated more than three standard deviations from a subject's mean in a given fade-in condition were excluded. By these criteria $6.6 \%$ and $4.4 \%$ of the latencies from the fade-in and instant conditions, respectively, were excluded; this difference was not significant $\left[F(1,19)=2.58, M S_{\mathrm{e}}=\right.$ 27.17].

Table 1 shows the mean word-naming latencies obtained in Experiment 1 . These latencies were analyzed with fade-in speed (instant, gradual) $\times$ word frequency (high, medium, low) $\times$ occurrence (first, second) repeated measures analyses of variance (ANOVAs). Since word frequency is a language variable (Clark, 1973), separate analyses were carried out with subjects as the random factor (referred to as $F_{1}$ ), and with items as the random factor (referred to as $F_{2}$ ). As expected, words that faded in gradually were named more slowly than were words appearing instantly $\left[F_{1}(1,19)=1,082.9, M S_{\mathrm{e}}=31,387.3\right.$; $\left.F_{2}(1,117)=7,393.4, M S_{\mathrm{e}}=9,288.3\right]$. The effect of word frequency on naming was also highly significant $\left[F_{1}(2,38)=28.3, M S_{\mathrm{e}}=1,679.2 ; F_{2}(2,117)=6.8\right.$, $\left.M S_{\mathrm{e}}=16,171\right]$, indicating that words of higher frequency were named faster than words of lower frequency. The effect of word frequency was greater for words that faded in gradually than for words appearing instantly, as is shown by a significant interaction between speed of presentation and word frequency $\left[F_{1}(2,38)=12.5, M S_{\mathrm{e}}=\right.$ $\left.1,051.8 ; F_{2}(2,117)=3.3, M S_{\mathrm{e}}=9,288.3\right]$. Words were named faster on their second occurrence than they were on their first occurrence $\left[F_{1}(1,19)=115.2, M S_{\mathrm{e}}=6,068.7\right.$; $\left.F_{2}(1,117)=433.6, M S_{\mathrm{e}}=3,124.5\right]$, showing that there was significant priming in the experiment. These priming effects are shown in Figure 2 and are analyzed next.

The priming data, displayed in Figure 2, were analyzed with 2 (fade-in speed: instant, gradual) $\times 3$ (word frequency: low, medium, high) ANOVAs. The magnitude of the priming effects was much larger when the words appeared gradually than when they appeared instantly $\left[F_{1}(1,19)=117.7, M S_{\mathrm{e}}=5,572.1 ; F_{2}(1,117)=337.7\right.$, $\left.M S_{\mathrm{e}}=3,715.9\right]$. Thus, slowing down the appearance of the words reduced baseline naming performance and also dramatically increased measured priming effects. Overall, the effect of word frequency on priming also reached significance $\left[F_{1}(2,38)=23.9, M S_{\mathrm{e}}=1,242.4\right.$;

Table 1

Naming Latencies (RTs, in Milliseconds, With Standard Deviations) Obtained in Experiment 1

\begin{tabular}{|c|c|c|c|c|c|c|c|c|}
\hline \multirow[b]{3}{*}{ Frequency } & \multicolumn{4}{|c|}{ Fade-In } & \multicolumn{4}{|c|}{ Instant } \\
\hline & \multicolumn{2}{|c|}{$\begin{array}{l}\text { First } \\
\text { Time }\end{array}$} & \multicolumn{2}{|c|}{$\begin{array}{l}\text { Second } \\
\text { Time }\end{array}$} & \multicolumn{2}{|c|}{$\begin{array}{l}\text { First } \\
\text { Time }\end{array}$} & \multicolumn{2}{|c|}{$\begin{array}{l}\text { Second } \\
\text { Time }\end{array}$} \\
\hline & RT & $S D$ & RT & $S D$ & RT & $S D$ & RT & $S D$ \\
\hline Low & $1,466.9$ & 156.9 & $1,236.9$ & 123.1 & 593.7 & 98.8 & 549.9 & 85.6 \\
\hline Medium & $1,396.1$ & 135.6 & $1,214.4$ & 139.7 & 569.7 & 90.7 & 543.6 & 90.5 \\
\hline High & $1,345.5$ & 110.1 & $1,211.4$ & 123.6 & 565.2 & 88.5 & 533.2 & 78.5 \\
\hline
\end{tabular}




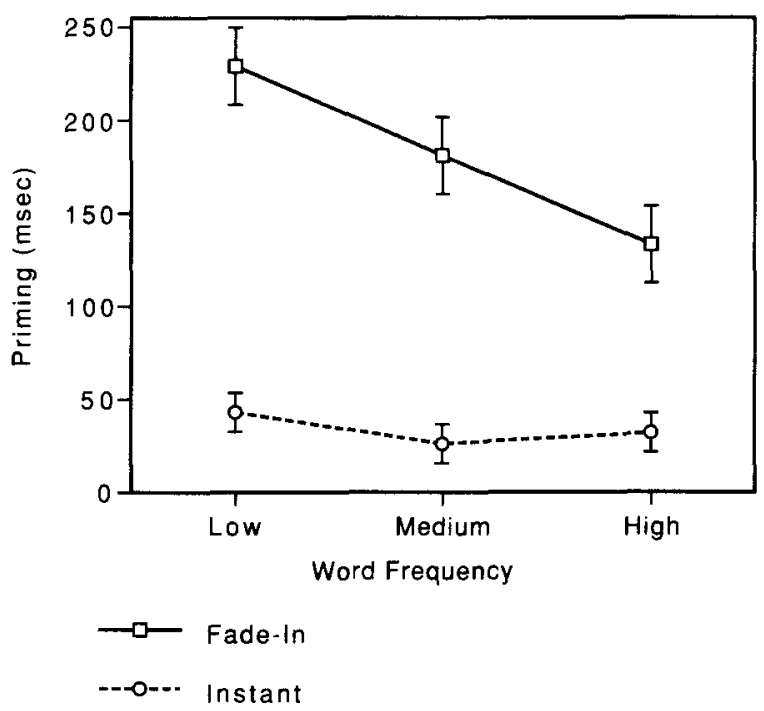

Figure 2. Priming effects obtained in Experiment 1 as a function of word frequency. In the instant condition, words were presented instantaneously. In the fade-in condition, each word appeared gradually over a 1.7-sec period. Error bars show within-subjects confidence intervals (Loftus \& Masson, 1994).

$F_{2}(2,117)=7.0, M S_{\mathrm{e}}=6,248.9 \mathrm{]}$. Most importantly, however, there was a highly significant interaction between fade-in speed and word frequency $\left[F_{1}(2,38)=13.0\right.$, $\left.M S_{\mathrm{e}}=1,396.2 ; F_{2}(2,117)=7.4, M S_{\mathrm{e}}=3,715.9\right]$, indicating that the effect of word frequency on priming was larger when the words appeared gradually than when they appeared instantly. Indeed, although the effect of word frequency on priming was highly significant when the words appeared gradually $\left[F_{1}(2,38)=21.9, M S_{\mathrm{e}}=\right.$ $\left.2,099.0 ; F_{2}(2,117)=8.6, M S_{\mathrm{e}}=7,863.9\right]$, the frequency effect was only marginally significant when the words appeared instantly $\left[F_{1}(2,38)=3.1, M S_{\mathrm{e}}=539.6, p=\right.$ $\left..057 ; F_{2}(2,117)=1.7, M S_{\mathrm{e}}=2,101\right]$.

Analysis of true naming errors (i.e., not including responses that were excluded because of extreme latencies) revealed only one significant effect-that a larger proportion of the responses in the fade-in condition $(5.5 \%$ errors) than in the instant condition ( $2.6 \%$ errors) were naming errors $\left[F(1,19)=4.8, M S_{\mathrm{e}}=4.1\right]$. Thus, the condition that resulted in the slowest naming responses also produced the most naming errors; there is, therefore, no evidence of a speed-accuracy tradeoff. Neither the main effects of occurrence and word frequency nor any interaction approached significance in the analyses of naming errors.

The manipulation of fade-in speed in the present experiment had two main consequences-it affected baseline naming speed and the magnitude of priming. Words that faded in gradually resulted in slower baseline naming speed and produced dramatically larger priming effects than did words appearing instantly. This clearly demonstrates that measured priming effects can be sig- nificantly affected by manipulating the availability of perceptual information from the presented stimuli. In the present case, increasing the difficulty level of the visual processing by degrading the stimuli resulted in an increased magnitude of priming effects.

The crucial finding in Experiment 1 is that manipulation of the speed of the appearance of the words also determined the extent to which the independent variable word frequency affected priming. When the words appeared instantly, the effect of word frequency on priming failed to reach significance. On the other hand, when the words appeared gradually, the effect of word frequency on priming was large and highly significant. Thus, within a single word-naming experiment, apparently conflicting findings - no frequency effect on priming in a wordnaming task and a significant effect of frequency on priming in lexical-decision tasks - have been replicated (Scarborough et al., 1977, 1979).

These results are consistent with the hypothesis that priming effects were relatively more constrained by high levels of baseline naming performance in the instant condition than they were in the fade-in condition.

According to the model outlined above, familiarity with the stimuli (the information available from prior encounters, $O$ ), stimulus clarity (the perceptual information available from the stimulus, $P$ ), and the information available from a specific prior study episode $(S)$ all affect the same stage of processing, and they should interact in determining identification performance. This is precisely what was observed in Experiment 1. A significant interaction between word frequency (familiarity) and fade-in speed (stimulus clarity) was observed in wordnaming performance. Priming effects were larger for lowfrequency than for high-frequency words, and greater priming effects were obtained when the words faded in gradually than when they occurred instantly. Finally, the frequency effect on priming was larger in the fade-in than in the instant condition. Most of these interactions have also been obtained in previous studies (see, e.g., Besner \& Swan, 1982; Jacoby \& Dallas, 1981; Norris, 1984; Scarborough et al., 1977). However, the interaction between word frequency and stimulus clarity in word identification was not observed in two early studies by Stanners, Jastrzembski, and Westbrook (1975) and Becker and Killion (1977). In a more recent study, Norris (1984), in agreement with the present findings, did obtain such an interaction. One possible reason for these discrepant findings is that the early studies lacked sensitivity, in that the stimulus clarity manipulations were not large enough to produce a significant interaction effect with word frequency. Across Becker and Killion's two experiments, the average effect of stimulus clarity was about $35 \mathrm{msec}$, and no interaction was observed with word frequency. In Stanners et al.'s experiment the effect of stimulus clarity was about $100 \mathrm{msec}$, and, although the interaction with frequency was not significant, there was a small effect in the expected direction. A larger stimulus-clarity effect 
of about $160 \mathrm{msec}$ on the first presentation of items (140 msec overall) was produced by Norris (1984), who also reported a significant stimulus-clarity by wordfrequency interaction. In the present experiment, the fade-in effect was about $820 \mathrm{msec}$ on the first presentation ( $750 \mathrm{msec}$ overall), and a highly significant interaction with word frequency was observed. Thus, the present finding does not appear to be in conflict with previous findings, and it supports the view that perceptual information, familiarity information, and specific study information interact in word identification.

\section{EXPERIMENT 2}

A surprisingly large increase in the magnitude of priming was observed in Experiment 1 when the mode of stimulus presentation was changed from instantly to gradually appearing words. The priming effects obtained in the fade-in condition were more than four times as large as those obtained in the instant condition (see Figure 2). This raises the question of whether it is possible to increase the magnitude of priming even further in the present naming task. Experiment 2 was carried out to address this question and to replicate and extend the findings of Experiment 1. In Experiment 2, fade-in times were increased to $1 \mathrm{sec}$ in the fast and $2.5 \mathrm{sec}$ in the slow fadein conditions.

A second important issue addressed in Experiment 2 is whether a relationship can be observed between priming and explicit-memory measures. In Experiment 1, it was found that the effect of word frequency on priming was significant only in the slow fade-in condition that produced the largest priming effects. It is possible that a relationship between priming and measures of explicit memory would also emerge as the magnitude of priming is increased. Experiment 2 investigates this issue by including tests of recognition memory for items employed in the word-naming task.

In word-stem or word-fragment completion tasks, stochastic independence has often been reported between measures of completion performance and measures of explicit memory (Light, Singh, \& Capps, 1986; Tulving et al., 1982). Contingency analysis of implicit- and explicit-memory measures has been criticized on many grounds (Hintzman, 1993; Hintzman \& Hartry, 1990; Ostergaard, 1992, 1995; Shimamura, 1985). One problem is that, in completion tasks, many stems or fragments are completed with target solutions, even when these solutions have not been studied. Furthermore, there is no way of distinguishing between target solutions that are produced because of prior study and those that simply reflect baseline completion performance. Therefore, only overall completion performance can be related to explicit memory in such studies. In other words, the reported contingency relations do not reflect relations between priming effects (i.e., the aspects of performance mediated by memory) and explicit-memory measures, but rather relations between overall performance in implicit- and explicit-memory tasks. This is a critical issue, because it is often the case that study (priming) effects are relatively small in implicit-memory tasks and, in many cases, too small to have a significant impact on the overall contingency relation (Ostergaard, 1992). The procedure used in the present investigation allows a measure of priming to be obtained for each item, namely, the difference between naming latency for the first and second occurrence of an item. It is therefore possible to determine directly the relationship between priming and recognition memory. However, such a relationship, even if it exists, may not be detectable when priming effects are constrained by high levels of baseline performance on the implicit-memory task. Consequently, in accordance with the general hypothesis of this investigation, it is expected that a relation between priming and recognition memory is more likely to be observed in the slow than in the fast fade-in condition.

\section{Method}

Subjects. Twenty University of California, San Diego, students participated in this experiment. They were paid at a rate of $\$ 8$ per hour. None of the subjects had participated in Experiment 1.

Design and Materials. The design and materials were identical to those of Experiment 1, with the following exceptions. A recognitionmemory test was administered after each block of word-naming trials. Because distractor (new) items were required for the recognitionmemory test, two additional matched word lists were constructed, each consisting of 20 low-, 20 medium-, and 20 high-frequency words, for use as distractor items. The two presentation conditions were a fast fade-in condition, in which each item was presented gradually over a period of $1 \mathrm{sec}$, and a slow fade-in condition, in which the items faded in over a 2.5 -sec period.

Procedure. The procedure was identical to that of Experiment 1 , except that, immediately after each block of word-naming trials (fast or slow fade in), a recognition memory test was given. In this test, the 60 critical items from the preceding word-naming task together with 60 matched new items were presented, one at a time, in the same random order to all subjects. The subject was instructed that half the words would have been read on the immediately preceding word-naming task and half would be new words. For each item, the subjects decided if the word was an old or a new word.

\section{Results and Discussion}

Recognition-memory performance was not affected by the speed with which the old items faded in during the naming task. Neither the factor fade-in speed nor any in-

Table 2

Proportions (With Standard Deviations) of Hit and False Positive Responses Obtained in Experiment 2

\begin{tabular}{|c|c|c|c|c|c|c|}
\hline \multirow[b]{3}{*}{ Response } & \multicolumn{6}{|c|}{ Word Frequency } \\
\hline & \multicolumn{2}{|c|}{ High } & \multicolumn{2}{|c|}{ Medium } & \multicolumn{2}{|c|}{ Low } \\
\hline & Prop. & $S D$ & Prop. & $S D$ & Prop. & $S D$ \\
\hline Hit & .84 & .11 & .89 & .08 & .91 & .08 \\
\hline False positive & .14 & .12 & .08 & .09 & .09 & .07 \\
\hline
\end{tabular}


teractions involving this factor approached significance (all $F \mathrm{~s}<1$ ). The analyses of recognition-memory performance are therefore reported for data collapsed across fade-in conditions.

A response type (hit, false positive) $\times$ word frequency ANOVA of the recognition-memory data shown in Table 2 revealed that significantly more hits than falsepositive responses were produced $[F(1,19)=587.6$, $\left.M S_{\mathrm{e}}=0.031\right]$. Overall, the effect of word frequency failed to reach significance $\left[F(1,19)=0.8, M S_{\mathrm{e}}=0.002\right]$, whereas the interaction between response type (hit vs. false positive) and word frequency was highly significant $\left[F(2,38)=22.0, M S_{\mathrm{e}}=0.002\right]$. The interaction reflects the fact that word frequency had opposite effects on hits and false-positive responses. This mirror effect is a well-established phenomenon in recognition-memory studies (Glanzer \& Adams, 1985). Significantly more hit responses were produced to low- $[t(19)=4.16]$ and medium- $[t(19)=2.96]$ frequency words than were produced to high-frequency words, whereas the difference between low- and medium-frequency words failed to reach significance $[t(19)=1.25]$. Significantly fewer falsepositive responses were produced to low- $[t(19)=3.23]$ and medium- $[t(19)=4.68]$ frequency distractors than were produced to high-frequency distractors, whereas again the difference between low- and medium-frequency items failed to reach significance $[t(19)=0.641]$.

By the criteria described above, $3.4 \%$ and $4.0 \%$ of the naming latencies were excluded from analyses in the fast and slow fade-in conditions, respectively. This difference did not approach significance $\left[F(1,19)<1, M S_{\mathrm{e}}=\right.$ $0.001]$. The mean naming latencies obtained in Experiment 2 are shown in Table 3 .

The pattern of word-naming data is similar to the pattern obtained in Experiment 1. As in Experiment 1, since the language variable word frequency was a factor in the experiment, the data were analyzed both with subjects as the random factor $\left(F_{1}\right)$ and with items as the random factor $\left(F_{2}\right)$. Word-naming latencies were longer in the slow fade-in condition than in the fast fade-in condition $\left[F_{1}(1,19)=1,170.5, M S_{\mathrm{e}}=32,354.8 ; F_{2}(1,117)=\right.$ $\left.5,233.3, M S_{\mathrm{e}}=14,601.2\right]$, and the effect of word frequency on naming was highly significant $\left[F_{1}(2,38)=\right.$ $\left.53.0, M S_{\mathrm{e}}=3,567.8 ; F_{2}(2,117)=9.4, M S_{\mathrm{e}}=43,478.8\right]$. With respect to overall naming latencies, the frequency by fade-in speed interaction reached significance only in the analysis with subjects as the random factor

Table 3

Naming Latencies (RTs, in Milliseconds, With Standard Deviations) Obtained in Experiment 2

\begin{tabular}{|c|c|c|c|c|c|c|c|c|}
\hline \multirow[b]{3}{*}{ Frequency } & \multicolumn{4}{|c|}{ Slow Fade-In } & \multicolumn{4}{|c|}{ Fast Fade-In } \\
\hline & \multicolumn{2}{|c|}{$\begin{array}{l}\text { First } \\
\text { Time }\end{array}$} & \multicolumn{2}{|c|}{$\begin{array}{c}\text { Second } \\
\text { Time }\end{array}$} & \multicolumn{2}{|c|}{$\begin{array}{l}\text { First } \\
\text { Time }\end{array}$} & \multicolumn{2}{|c|}{$\begin{array}{c}\text { Second } \\
\text { Time }\end{array}$} \\
\hline & RT & $S D$ & RT & $S D$ & RT & $S D$ & RT & $S D$ \\
\hline Low & $2,012.8$ & 184.3 & $1,684.1$ & 127.3 & $1,108.0$ & 107.9 & 946.2 & 83.7 \\
\hline Medium & $1,901.8$ & 171.1 & $1,620.5$ & 119.7 & $1,042.9$ & 83.6 & 913.0 & 71.3 \\
\hline High & $1,836.9$ & 151.7 & $1,629.3$ & 140.7 & $1,008.8$ & 91.5 & 899.6 & 75.2 \\
\hline
\end{tabular}

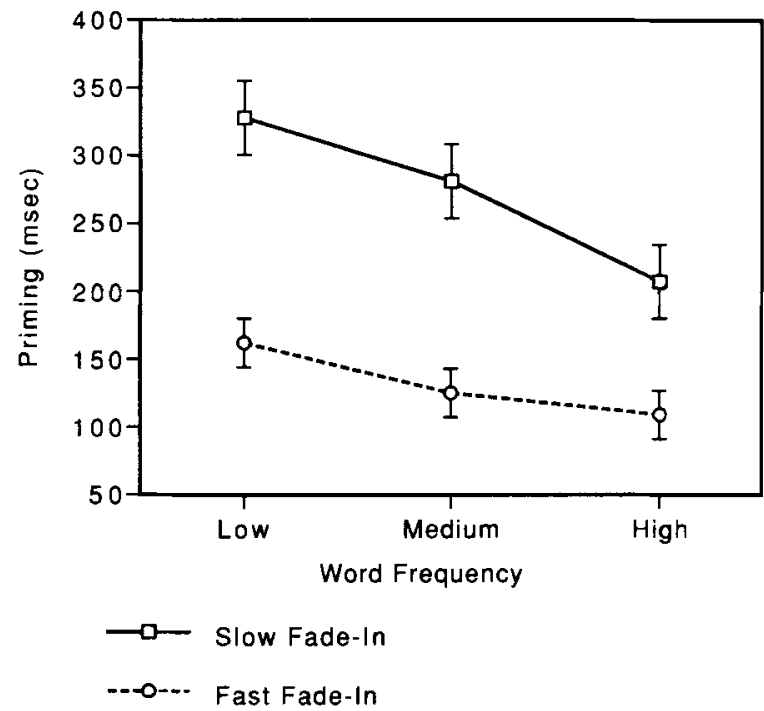

Figure 3. Priming effects obtained in Experiment 2 as a function of word frequency. In the fast and slow fade-in conditions, each word appeared gradually over periods of $1 \mathrm{sec}$ and $2.5 \mathrm{sec}$, respectively. Error bars show within-subjects confidence intervals (Loftus \& Masson, 1994).

$\left[F_{1}(2,38)=4.33, M S_{\mathrm{e}}=2,528.7 ; F_{2}(2,117)=1.9\right.$, $\left.M S_{\mathrm{e}}=14,601.2\right]$, suggesting that the frequency effect was greater in the slow than in the fast fade-in condition. As in Experiment 1, highly significant priming effects were observed; words were named faster on their second than on their first occurrence $\left[F_{1}(1,19)=258.7, M S_{\mathrm{e}}=\right.$ $\left.9,563.9 ; F_{2}(1,117)=735.0, M S_{\mathrm{e}}=6,608.2\right]$. These priming effects are shown in Figure 3.

A fade-in condition $\times$ word frequency repeated measures ANOVA of the priming data obtained in Experiment 2 showed that priming effects were significantly larger in the slow than in the fast fade-in condition $\left[F_{1}(1,19)=65.6, M S_{\mathrm{e}}=9,042.3 ; F_{2}(1,117)=116.7\right.$, $\left.M S_{\mathrm{e}}=9,693.1\right]$. The effect of word frequency on priming was also significant $\left[F_{1}(2,38)=24.1, M S_{\mathrm{e}}=3,125.3\right.$; $\left.F_{2}(2,117)=11.6, M S_{\mathrm{e}}=13,216.3\right]$, and, most importantly, the interaction between fade-in speed and word frequency was significant $\left[F_{1}(2,38)=6.6, M S_{\mathrm{e}}=\right.$ $\left.2,075.1 ; F_{2}(2,117)=3.1, M S_{\mathrm{e}}=9,693.1\right]$. Thus, as in Experiment 1 , the effect of word frequency on priming was greater in the slow fade-in condition than in the fast fade-in condition. In the present experiment, the frequency effect was significant in both the slow fade-in condition $\left[F_{1}(2,38)=20.5, M S_{\mathrm{e}}=3,636.9 ; F_{2}(2,117)=\right.$ $\left.7.9, M S_{\mathrm{e}}=19,403.4\right]$ and the fast fade-in condition $\left[F_{1}(2,28)=9.3, M S_{\mathrm{e}}=1,563.5 ; F_{2}(2,117)=8.8\right.$, $\left.M S_{\mathrm{e}}=3,506.1\right]$. In the slow fade-in condition, lowfrequency words produced significantly more priming than did medium- $[t(19)=3.57]$ and high- $[t(19)=$ 5.61 ] frequency words, and medium-frequency items produced more priming than did high-frequency words $[t(19)=3.48]$. In the fast fade-in condition, more priming was similarly observed with low-frequency words 
than with medium- $[t(19)=3.05]$ and high- $[t(19)=$ 3.48] frequency words, whereas in this case the difference between medium- and high-frequency items failed to reach significance $[t(19)=1.58]$.

Analysis of naming errors ( $1 \%$ of the responses) in the present experiment revealed no significant effects.

Next, the relationship between priming and recognition-memory measures is considered. The correlations across subjects between the average priming obtained with the target items in the naming tasks and the proportion of hits produced for these same items in the recognition tasks are first examined. In the fast fade-in condition, the correlation between the amount of priming and the proportion of hits failed to reach significance $[r=$ $.37, t(18)=1.69$ ]. However, in the slow fade-in condition, in which priming effects were larger, the correlation between priming and recognition memory was higher and did reach significance $[r=.45, t(18)=2.14]$. Thus, when priming effects are large, a direct relationship between priming and recognition memory may be observed.

A second way in which to assess the relationship between priming and recognition memory is to determine whether the items that were correctly recognized (hits) produced more priming in the word-naming task than did the items that were missed on the recognitionmemory test. Figure 4 shows, for the slow and fast fadein conditions, the amount of priming obtained in the naming task for words that were correctly recognized and words that were missed in the recognition-memory test. A fade-in speed $\times$ hit versus miss responses ANOVA of these data revealed significant effects of both the main factors-fade-in speed $\left[F(1,19)=31.8, M S_{\mathrm{e}}=7,513.7\right]$ and hit versus miss responses $\left[F(1,19)=7.22, M S_{\mathrm{e}}=\right.$ $5,580.5$ - as well as a significant interaction $[F(1,19)=$

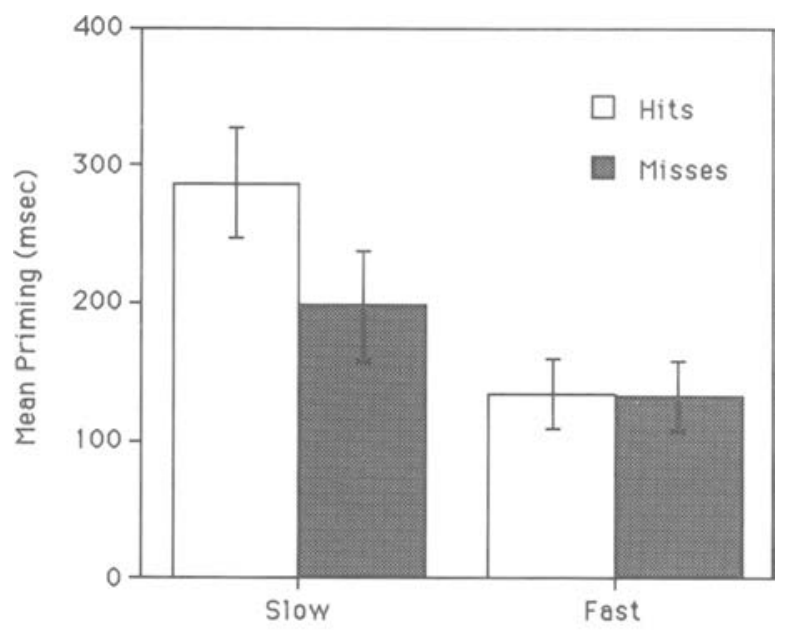

Fade-In Speed of Presented Words

Figure 4. Priming effects for items that were correctly recognized and items that were missed on the recognition-memory tests in Experiment 2. Error bars show within-subjects confidence intervals (Loftus \& Masson, 1994).
8.29, $\left.M S_{\mathrm{e}}=4,612.9\right]$. The main effect of fade-in speed simply reflects the fact that larger priming effects were obtained in the slow than in the fast fade-in conditions. The significance of the factor hit versus miss responses indicates that the words that were correctly recognized on the recognition-memory test had produced significantly more priming on the preceding naming task than had words that were missed on the recognition-memory test. However, the significant interaction reflects the fact that, whereas in the slow fade-in condition, words that were correctly recognized on the recognition-memory test had produced significantly more priming on the preceding naming task than had words that were missed on the recognition-memory test $\left[F(1,19)=10.7, M S_{\mathrm{e}}=\right.$ $7,323.8]$, this difference between hits and misses did not approach significance in the fast fade-in condition $\left[F(1,19)<1, M S_{\mathrm{e}}=2,869.6\right]$. Thus, as in the correlational analysis, a relationship between magnitude of priming and recognition-memory performance could be observed in the condition that produced the largest priming effects (i.e., slow fade in).

The manipulation of fade-in speed of the stimuli in this experiment, as in Experiment 1, affected both baseline naming performance, and the magnitude of priming effects. Average priming effects that were obtained in the four fade-in conditions investigated in Experiments 1 and 2 are shown in Figure 5. Clearly, the fade-in manipulations had dramatic effects on measured priming. Over the range of fade-in conditions investigated (from instant to $2.5 \mathrm{sec}$ ), the magnitude of measured priming increased by more than a factor of eight.

An important finding is that fade-in speed also determined the extent to which the independent variable word frequency affected the magnitude of priming. If only the difference between low- and high-frequency words is considered, then the word-frequency effect on priming increased from an insignificant $12 \mathrm{msec}$ in the instant condition of Experiment 1 to a large and highly significant $121 \mathrm{msec}$ effect in the slowest fade-in condition of Experiment 2 . In other words, as the magnitude of priming was increased, the effect of word frequency on priming also increased. If baseline naming performance is taken as an index of task difficulty, then it can be concluded that, in the present task, increasing task difficulty resulted in an increased magnitude of priming and in an increased effect of the independent variable word frequency on priming.

In Experiment 2, a direct relationship between priming and explicit-memory performance could be observed in the slow fade-in condition. Ostergaard (1992) showed how the previous failure to find significant dependence between priming and explicit-memory measures could be a consequence of priming effects being relatively small in comparison with overall levels of performance. Furthermore, contingency analyses of implicit- and explicitmemory measures have always been carried out on overall performance with previously studied items in the implicit-memory task. In other words, no distinction has been made between baseline performance and priming 


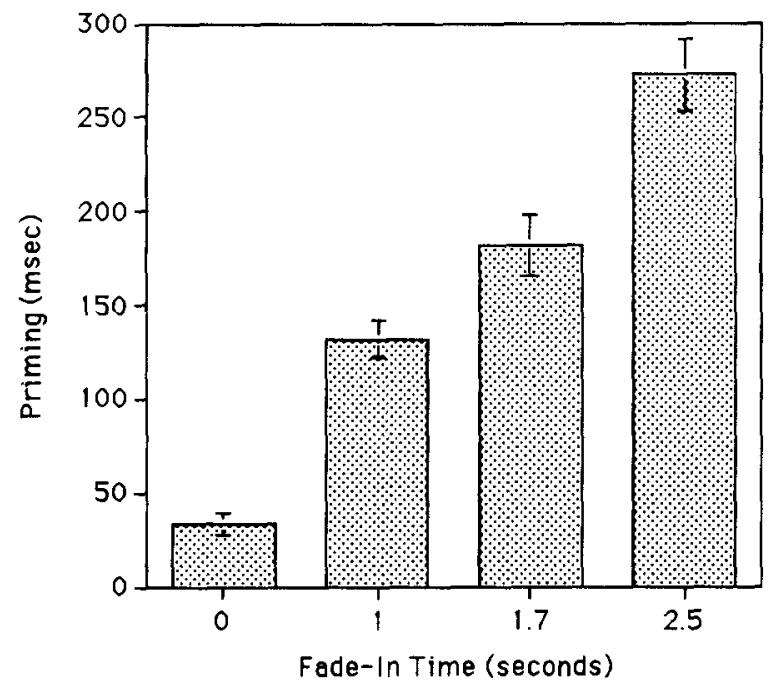

Figure 5. Magnitude of priming effects across the four fade-in conditions investigated in Experiments 1 and 2. Error bars show standard error of the means, because this is a comparison across different experiments.

in such analyses. In the present experiment, actual priming effects were directly compared with recognition-memory measures. Under those circumstances, a direct relationship between priming and explicit-memory performance could be observed in the condition producing the largest priming effects. The hypothesis of the present investigation is that, when the task is easy and subjects achieve high levels of baseline performance, the possible magnitude and variability of priming effects are constrained. Relatively familiar items presented clearly produce high levels of baseline identification performance, which indicates that, even for nonstudied items, the perceptual information and the information available in memory from prior encounters with the items are sufficient for the subjects to carry out the task very efficiently. Under such circumstances, the information that is available from a specific prior study episode may affect performance only to a very limited extent, and the size of priming effects may not directly reflect the amount of information that is available from the prior study episode. Consequently, it is not surprising that no relationship is observed between the magnitude of priming and explicit-memory measures when priming is constrained. The important finding is that, when the constraints are reduced, a relationship between priming and explicit memory becomes detectable. The present finding is consistent with previous reports of a relationship between implicit- and explicit-memory measures (Feustel et al., 1983; Ostergaard, 1994; Perruchet \& Baveux, 1989).

\section{EXPERIMENT 3}

As discussed in the general introduction, two factors that have produced variable, and sometimes conflicting, results in priming studies are delay of the priming test and the number of repetitions of the primed items. Experiment 3 was designed to address these issues within the framework of the fade-in procedure. Priming effects are investigated after one to four repetitions and after short and long delays, under two fade-in conditions.

According to the general hypothesis of the present investigation, delay and repetition should not affect priming when items are presented instantaneously, whereas an effect of both of these factors is expected when the items fade in gradually.

\section{Method}

Subjects. Twenty University of California, San Diego, students participated in this experiment. They were paid at a rate of $\$ 8$ per hour.

Materials and Design. Four target-word lists were constructed, each consisting of 15 words ( 2 four-letter words, 3 five-letter words, 4 six-letter words, 3 seven-letter words, and 3 eight-letter words). The mean (Kučera \& Francis, 1967) frequency count for each list was 24.2 (range: 5-75). The same two presentation conditions that were employed in Experiment 1 were investigated in this experiment - words appearing instantly and words fading in over a period of $1.7 \mathrm{sec}$. Two target-word lists were used in each fade-in condition. One list was presented four times during the first part of the experiment (the repeated list). The other list was employed to assess the effect of delay on priming (the delay list), and this list was presented once during the first part of the experiment and then again after a delay of $45 \mathrm{~min}$. The four target lists were rotated over conditions between subjects, such that each list was used equally often in each condition. Additionally, 70 words matching the target words with respect to length and frequency were selected as filler words.

Two blocks of 100 naming trials were constructed, one for each fade-in condition. Each block consisted of four sub-blocks of 25 trials. In each sub-block, the 15 words from the repeated list were presented together with 10 other words in a pseudorandom order, with the restriction that no more than 3 successive words could be from the repeated list. The 10 other words were a combination of words from the delay list and filler words. The first 5 words in each main block of 100 trials were filler words. Thus, over the course of a block of 100 trials, the words from the repeated list were presented four times each, and the words from the delay list were presented once each. Additionally, two delay blocks were constructed, one for each of the fade-in conditions. Each delay block of 28 trials consisted of the 15 words from the delay list plus 13 filler words presented in random order, with the restrictions that the first 3 words were filler (practice) words and that no more than 3 words from the delay list could occur successively.

Procedure. The method of stimulus presentation and response recording was the same as that described above. First, the subject was given the two blocks of 100 -word naming trials. The order of instant and fade-in conditions was balanced across subjects. Before each block, the subject was informed whether words in that block would be presented instantly or would fade in gradually. As in the previous experiments, the subjects were instructed to name each word as quickly as possible without making errors. Subjects were informed that some words would be repeated several times.

After the subject had completed the first two naming blocks of trials (instant and fade-in), there was a 45-min delay, during which the subject participated in other experiments. There was no overlap between the materials used in the present experiment and the tasks carried out during the delay interval. After the 45 -min delay, the subject was given the final two blocks (delay blocks) of 28 naming trials each. For each subject, the order of instant and fade-in conditions at the delayed test was the same as in the first two blocks of trials. 
Table 4

Naming Latencies (RTs, in Milliseconds, With Standard Deviations) for the First to the Fourth Occurrence of Repeated Items in Experiment 3

\begin{tabular}{|c|c|c|c|c|c|c|c|c|}
\hline & & & & Occu & ence & & & \\
\hline & Fir & & $\mathrm{Sec}$ & & Thi & & & \\
\hline & RT & $S D$ & RT & $S D$ & RT & $S D$ & RT & $S D$ \\
\hline nsta & 545 & 53 & 523 & 54 & 522 & 50 & 525 & 60 \\
\hline Fade-in & 1,211 & 91 & 1,029 & 72 & 1,003 & 79 & 975 & 73 \\
\hline
\end{tabular}

\section{Results and Discussion}

By the exclusion criteria described above, $4.7 \%$ and $4.9 \%$ of the naming responses were excluded from analyses in the instant and fade-in conditions, respectively. This difference did not approach significance $[F(1,19)<1$, $\left.M S_{\mathrm{e}}=8.6\right]$.

First, the effect on naming speed of up to four presentations of the target items will be analyzed; then the persistence of priming over the delay interval will be considered. Mean naming latencies for each of the four occurrences of repeated items are shown in Table 4 for the two fade-in conditions.

A fade-in speed (instant and gradual) $\times$ occurrence (1-4) within-subjects ANOVA revealed, as expected, that naming was slower in the fade-in than in the instant condition $\left[F(1,19)=928.9, M S_{\mathrm{e}}=11,881.9\right]$. The factor occurrence $\left[F(3,57)=77.7, M S_{\mathrm{e}}=1,761.7\right]$ and, importantly, the interaction $\left[F(3,57)=64.7, M S_{\mathrm{e}}=1,438.5\right]$ were also significant. The significant interaction indicates that the effect of repetition was greater in the fadein than in the instant condition. Two further analyses were carried out in order to address this issue. First, only the data from the first two occurrences were analyzed in order to assess the standard priming effects from a single prior encounter with the primed items. Significant priming was observed in both the instant condition $\left[F(1,19)=10.4, M S_{\mathrm{e}}=446.7\right]$ and the fade-in condition $\left[F(1,19)=137.5, M S_{\mathrm{e}}=2,408.3\right]$. However, as in the previous experiments, the priming effect was significantly larger in the fade-in than in the instant condition $\left[F(1,19)=87.7, M S_{\mathrm{e}}=1,466.5\right]$. Next, the naming latencies from the second through the fourth occurrence were analyzed in order to determine whether priming was further augmented by additional repetitions. In the fade-in condition, the effect of additional repetitions on naming was highly significant $\left[F(2,38)=9.7, M S_{\mathrm{e}}=\right.$ 1,513.4], whereas in the instant condition, there was no hint of an effect of additional repetitions $[F(2,38)<1$, $\left.M S_{\mathrm{e}}=387.1\right]$. Thus, significant priming effects were observed in both conditions, but a significant repetition effect was observed only in the fade-in condition.

The effect of delay on priming is considered next. Priming effects after short and long delays are shown in Figure 6. Short-delay priming is the difference between naming latencies at the first and second occurrences of the items that were repeated within each main block of 100 trials (average delay $=100 \mathrm{sec}$ ). Long-delay priming is the difference between naming latencies to items in the delay list at their first (and only) occurrence in the main block of 100 trials and their second occurrence after a 45-min delay in the delay block.

All priming effects shown in Figure 6 are significant $[t(19) \mathrm{s}>3.2]$. A presentation (instant, fade-in) $\times$ delay (short, long) within-subjects ANOVA of the priming data revealed significantly greater priming in the fade-in condition than in the instant condition $[F(1,19)=61.2$, $\left.M S_{\mathrm{e}}=4,590.0\right]$. Overall, the effect of delay was also significant $\left[F(1,19)=6.7, M S_{\mathrm{e}}=3,292.4\right]$. However, the important finding is that the interaction between presentation condition and delay is highly significant $[F(1,19)=$ 15.6, $\left.M S_{\mathrm{e}}=2,242.5\right]$. This interaction reflects the fact that, in the instant condition, there was no hint of an effect of the length of delay on priming $\left[F(1,19)<1, M S_{\mathrm{e}}=\right.$ $1,237.7]$, whereas in the fade-in condition, significantly less priming was observed after the long than after the short delay $\left[F(1,19)=13.1, M S_{\mathrm{e}}=4,297.2\right]$.

Overall, $2.3 \%$ of the responses in this experiment were naming errors. Analysis of the naming errors revealed only one significant effect, namely of occurrence $[F(3,57)=$ 3.1, $M S_{\mathrm{e}}=14.9$, which indicates that fewer naming errors occurred as the items were repeated more times (naming errors: first occurrence $=3 \%$, second occurrence $=$ $2.7 \%$, third occurrence $=1.5 \%$, fourth occurrence $=$ $1 \%)$.

This experiment produced results that were similar to several of the conflicting earlier findings with respect to the effects of delay and spaced repetitions on priming. Despite the fact that significant priming effects were obtained in the instant-presentation condition, neither delay nor up to four repetitions of the primed items affected priming in this condition. However, both of these variables significantly affected the magnitude of priming in the fade-in condition. This provides further support for the hypothesis that, when words are presented instantly

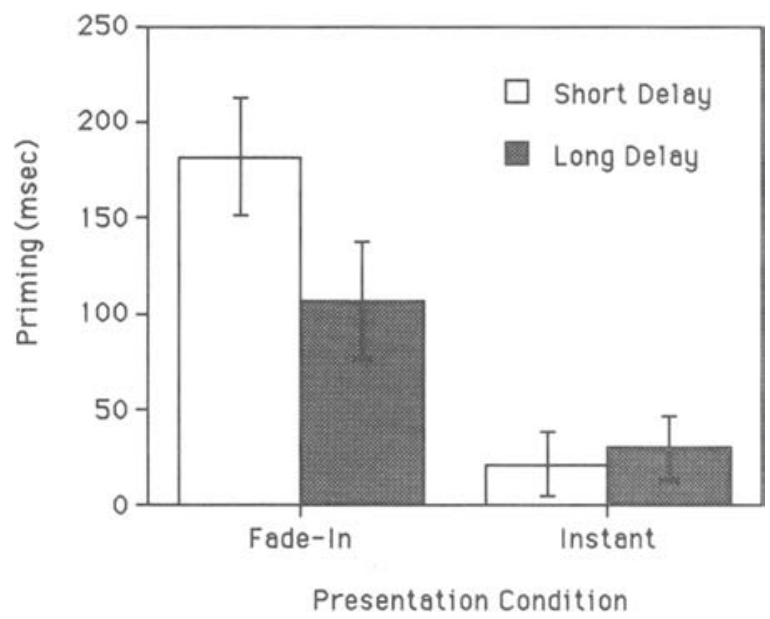

Figure 6. Priming effects obtained after short (approximately $100 \mathrm{sec}$ ) and long ( $45 \mathrm{~min}$ ) delays in Experiment 3. Error bars show within-subjects confidence intervals (Loftus \& Masson, 1994). 
for naming, priming effects are constrained and, therefore, cannot reflect variability in the availability of information from a specific prior study episode.

\section{EXPERIMENT 4}

The first three experiments have demonstrated that priming effects can be increased substantially by increasing the difficulty of the processing task that is being used to measure priming. Furthermore, as task difficulty, and consequently priming effects, are increased, the effects of several independent variables on priming are also increased. However, since the previous experiments employed a continuous-naming paradigm, in which items were presented the same way (slow fade-in, fast fade-in, or instant) at both the first and subsequent occurrences, it is not clear whether the increase in priming is due to a study effect or to a retrieval effect. It is possible that, when the items fade in slowly, the naming task requires more effort than it does when they appear quickly, and that this increased effort results in a stronger memory trace. On the other hand, it is also possible, as suggested by Ostergaard (1994), that it is the characteristics of the retrieval task that determine the size of measured priming effects. In that case, a memory trace of a given strength would have a greater effect on a difficult than on an easy processing task. This final experiment addresses these questions by investigating all four possible combinations of instant and fade-in presentation of items in study and test phases. If the effect of speed of presentation on priming is due to encoding differences, then fade-in study conditions should result in greater priming effects than should instantaneous presentation of words at study. On the other hand, if the important factor is the nature of the processing task at test, then the magnitude of priming should be greater when the test phase involves fade-in presentation than when it involves instant presentation of the primed items.

\section{Method}

Subjects. Twenty-four University of California, San Diego, students participated in this experiment. They were paid at a rate of $\$ 8$ per hour.

Materials and Design. The experiment consisted of four pairs of study-test phases. The design was a factorial combination of fade-in speed during the study phase (instant and fade-in) and fadein speed during the test phase (instant and fade-in). A repeated measures design was employed such that all subjects were tested in all four conditions. The order of the conditions, however, was rotated across subjects. As in Experiments 1 and 3, the two fade-in speeds were instantly appearing words and words appearing gradually over a period of $1.7 \mathrm{sec}$.

Eight matched target word lists were constructed, each consisting of 15 words of the following lengths: 1 four-letter word, 4 fiveletter words, 4 six-letter words, 3 seven-letter words, and 3 eightletter words. The mean Kučera and Francis (1967) frequency count for each list was 24.7 (range: 5-77). Two word lists were used in each of the four conditions of the experiment. The words from the first list (old words) were presented during the study phase and were presented again, randomly mixed with the words from the second list (new words), during the test phase. Three filler words were added to the beginning and the end of each study block of trials and to the beginning of each test block of trials. The assignment of list pairs to experimental conditions was rotated across subjects, such that a given pair of lists was used equally often in each of the four conditions. Furthermore, the lists employed as old words for half the subjects were used as new words for the remaining half of the subjects, and vice versa. In short, the experiment consisted of a series of four study and test phases. In each study phase, 21 words were presented, consisting of the 15 old words and 6 filler words. During the following test phase, 3 filler words were first presented, followed by the 15 old and 15 new words, presented in random order.

Procedure. The method of stimulus presentation and response recording was as described above. Subjects were instructed that a series of words would be presented and that they should name aloud each word as quickly as possible without making errors. They were told that some words would be repeated over the course of the experiment. Subjects were informed that some words would be presented instantaneously and some would gradually fade in on the computer screen. Immediately prior to each study or test block of trials, the subject was informed whether the words in that block would appear instantly or gradually.

\section{Results and Discussion}

By the usual criteria, $2.9 \%$ and $3.0 \%$ of the naming responses were excluded from analyses in the instant and fade-in conditions, respectively. This difference did not approach significance $\left[F(1,23)<1, M S_{\mathrm{e}}=5.2\right]$. As expected, overall naming latencies were significantly longer when the words appeared gradually (mean RT = $1,223 \mathrm{msec}$ ) than when they appeared instantly (mean $\mathrm{RT}=495 \mathrm{msec})\left[F(1,23)=1,137.8, M S_{\mathrm{e}}=22,300.7\right]$. In the test phases, previously studied (old) words were named significantly faster than new words showing that significant priming was obtained in the experiment [mean RT old $=826 \mathrm{msec}$, mean RT new $=892 \mathrm{msec}, F(1,23)=$ $\left.61.4, M S_{\mathrm{e}}=3,363.3\right]$. However, the data of interest in the present experiment are the priming effects obtained in the four conditions; these are shown in Table 5.

Priming effects were significant in all experimental conditions $[t(23) \mathrm{s}>2.5]$. A fade-in speed at study $\times$ fadein speed at test ANOVA of the priming data in Table 5 revealed a significant effect of fade-in speed at test $[F(1,23)$ $\left.=54.4, M S_{\mathrm{e}}=3,882.6\right]$, which indicates that greater priming effects were obtained when the words faded in gradually than when they appeared instantly during the test phase. The effect of fade-in speed during study just failed to reach significance $\left[F(1,23)=2.9, M S_{\mathrm{e}}=1,698.1\right]$, and

Table 5

Baseline Naming Latencies and Mean Priming Effects (RTs, in Milliseconds, With Standard Deviations) Obtained in Experiment 4

\begin{tabular}{|c|c|c|c|c|c|c|}
\hline & & \multicolumn{4}{|c|}{ Study Condition } & \multirow[b]{3}{*}{ Mean } \\
\hline \multirow{2}{*}{\multicolumn{2}{|c|}{$\begin{array}{c}\text { Test } \\
\text { Condition }\end{array}$}} & \multicolumn{2}{|c|}{ Instant } & \multicolumn{2}{|c|}{ Fade-In } & \\
\hline & & RT & $S D$ & RT & $S D$ & \\
\hline \multirow[t]{2}{*}{ Instant } & baseline & 508 & 88 & 501 & 71 & 504.5 \\
\hline & priming & 18 & 34 & 19 & 25 & 18.5 \\
\hline \multirow[t]{2}{*}{ Fade-in } & baseline & 1,278 & 135 & 1,279 & 175 & 1.278 .5 \\
\hline & priming & 99 & 64 & 126 & 92 & 112.5 \\
\hline \multicolumn{2}{|c|}{ Mean priming } & \multicolumn{2}{|c|}{58.5} & \multicolumn{2}{|c|}{72.5} & \\
\hline
\end{tabular}


the interaction did not approach significance $[F(1,23)=$ $\left.1.9, M S_{\mathrm{e}}=2,004.5\right]$.

Overall, $2.1 \%$ of the responses produced in this experiment were naming errors; however, statistical analysis of these errors revealed no significant effects.

The results of this experiment show clearly that the manipulation of task difficulty in the test phase has a large effect on the magnitude of measured priming. On average, the priming effects obtained when the items faded in gradually in the test phase were more than six times as large as those obtained when the words were presented instantaneously in the test phase. The effect of speed of presentation during the study phase failed to reach significance. Numerically, however, there was a small tendency toward larger priming effects after the fade-in than after the instantaneous study conditions, and it remains an open question whether this small effect on priming is real or due to experimental error. In a similar clarification task, Jacoby, Baker, and Brooks (1989) obtained a small but just significant effect of study condition on the identification of pictures that were gradually clarified. Pictures that were gradually clarified during study required slightly fewer steps of clarification to be identified in a subsequent test than did pictures that were presented instantaneously during the study phase. In Jacoby et al.'s experiment, the clarification occurred in discrete steps, and the rate of clarification was selfpaced by the subject. At each discrete level of degradation (clarification), the subject attempted to name the picture; when he or she either gave up or produced an incorrect name, he or she pressed a key on a computer keyboard in order to initiate the presentation of the next, more clarified, version of the picture. It is possible that transferappropriate processing played a larger role in Jacoby et al.'s task than it did in the present task. Identification of a stimulus at a given level of degradation may selectively benefit from the subject having encountered that stimulus in the same, or a very similar, degraded form. Jacoby et al. did not manipulate the mode of presentation at test; the stimuli were all gradually clarified, and there is, therefore, no way of knowing if such a retrieval-task manipulation would have produced a very large effect, as was the case in the present experiment. Nevertheless, the present results strongly support Ostergaard's (1994) suggestion that the characteristics of the retrieval task in priming studies can to a large extent determine the magnitude and pattern of priming obtained in an information-processing task.

\section{GENERAL DISCUSSION}

In the four experiments of the present investigation, a relatively simple manipulation of the speed with which stimuli fade in (are gradually clarified) in a word-naming task had dramatic consequences for performance and priming measures. Slowing down the appearance of the stimuli, not surprisingly, had a detrimental effect on baseline naming speed. However, this decrement in baseline naming performance was associated with a very substantial increase in the magnitude of priming effects obtained in the naming task. In Experiment 4, the priming effects obtained in the fade-in conditions were more than six times as large as those obtained in the instant conditions (see Table 5), and, over the four fade-in conditions investigated in Experiments 1 and 2, there was more than an eightfold increase in the magnitude of priming (see Figure 5). These large changes in priming effects were not produced by manipulating the encoding of information into memory; they occurred as a consequence of variations in the information-processing requirements of the retrieval task. In Experiment 4, closely matched items studied under identical conditions produced substantially larger priming effects when the retrieval task involved items that faded in gradually than when the items appeared instantly.

An important finding of this study relates to the effects of independent variables on measured priming. None of the experimental variables investigated (word frequency, spaced repetition, and delay) affected priming significantly in the instant conditions, in which priming effects were relatively small. However, all of these factors affected the magnitude of priming when the items faded in gradually and priming effects were relatively large. Thus, with this simple fade-in manipulation, it is possible to make the effects on priming of word frequency, spaced repetition, and delay appear and disappear at will. It should be noted that the failure to find effects of any of these experimental manipulations in the instant conditions is not a consequence of a lack of statistical power. In all cases, the power to detect in the instant condition an effect of the size obtained in the fade-in condition was greater than .98 .

Experimental dissociations between explicit and implicit measures of memory are often regarded as evidence for the existence of independent explicit and implicit memory systems (see, e.g., Squire, 1992; Tulving $\&$ Schacter, 1990). The present results weaken this evidence because clear dissociations between priming (implicit) measures were produced as a function of how rapidly the stimuli were presented. The dissociations between the fade-in conditions in the present investigation seem as convincing as dissociations that have been reported between explicit- and implicit-memory measures. To account for the present results in terms of a memorysystems model, it would seem necessary to postulate that priming in word naming was mediated by different memory systems in the different fade-in conditions. A distinction between perceptual and conceptual processing has been invoked to account for the divergent results obtained in different memory tasks (Jacoby, 1983b; Roediger, Weldon, \& Challis, 1989). However, an account of the present data in such terms also runs into difficulties, because the identification of single words out of context is considered by most to rely mainly on perceptual (data- 
driven) processing, regardless of whether the words are presented in an intact or a visually degraded form (Roediger \& McDermott, 1993).

\section{The "Contamination" Issue}

It could be argued that, in the fade-in conditions, the subjects had more time to use a conscious retrieval strategy - that is, to attempt to retrieve previously presented words-and that this putative use of explicit memory led to an apparent increase in priming. This explanation is implausible for several reasons. Even in the fade-in conditions of Experiments 1, 3, and 4, average response latencies were less than $1.5 \mathrm{sec}$. If that is enough time to allow explicit retrieval to inflate priming effects significantly, then priming effects obtained in tasks in which speeded responses are not required would be invalid. In tasks such as word-fragment completion and tachistoscopic identification, response times are typically longer than $1.5 \mathrm{sec}$ (see, e.g., Nelson, McEvoy, \& Bajo, 1988). Indeed, in word-fragment completion, average response latencies of from 3 to $6 \mathrm{sec}$ are common (Hayman \& Tulving, 1989). Similarly, long response times must occur in tasks that require subjects to try to identify items that are presented in a series of progressively less visually degraded representations of each item (see, e.g., Jacoby et al., 1989; Snodgrass \& Feenan, 1990; Warrington \& Weiskrantz, 1968).

Thus, the fade-in conditions of the present experiments do not appear to allow more time for conscious retrieval than is typically the case in priming experiments. Furthermore, it is not clear how the use of conscious retrieval strategies could improve performance in the present word-naming task. Even assuming that a subject has perfect recall of all previously presented words, the subject has no way of knowing which specific word will come up on the next trial. Only the first naming response given on each trial was accepted-that is, a subject was not allowed to produce multiple guessing responses in trying to name an item. Therefore, it is very unlikely that the correct item would be the first item recalled by a subject on a given trial. Indeed, on trials with nonrepeated words, a strategy of consciously recalling previously presented items would prompt an incorrect response. In other words, such a strategy would probably result in an unacceptably large number of naming errors.

The observed word-frequency effects are also inconsistent with the conscious-retrieval hypothesis. It is well established that high-frequency words are better recalled than low-frequency words (Gregg, 1976; Hall, 1954). Therefore, if the increase in priming effects in the fadein condition was due to subjects consciously recalling previously presented words, then priming effects for highfrequency words would be expected to be larger than priming effects obtained with low-frequency words. The opposite pattern was found in the present experiments; low-frequency words produced larger priming effects than did high-frequency words, and the magnitude of this fre- quency effect was greater in the fade-in condition than in the instant condition. In other words, the frequency effect on priming is in the opposite direction from what would be predicted from the conscious-recall hypothesis.

It might be argued that conscious-recognition memory for items may have augmented priming effects, and because recognition memory is better for low-frequency items than for high-frequency items (see, e.g., Glanzer \& Adams, 1985), such contamination could have produced the pattern of results observed here. However, it is not clear how recognition memory could affect identification performance, since presumably recognition memory for an item occurs after the subject has accessed the item's lexical representation and thereby identified it. It does not seem plausible that the subject could have consciousrecognition memory for an item that has not been identified. It is possible that, before the subject has identified a degraded item, candidates may come to mind, and if such a candidate is recognized as an old word, it may be produced as the identification response. In that way conscious recognition could affect identification performance. However, since priming is often regarded as being a significant determinant of the words that will come to mind when trying to interpret a stimulus, such an influence on identification performance may be regarded as partly reflecting priming. Furthermore, it seems that reliance on a strategy of conscious recognition of candidates that come to mind would lead to many naming errors. The particular old word that comes to mind and is recognized, on a given trial, may not be the correct one for identification. As in the case of conscious recall discussed above, relying on a strategy of recognition memory for candidate solutions to the degraded stimuli would most likely lead to incorrect identification responses for nonrepeated items.

Conscious recognition memory for studied items clearly occurs in priming tasks inasmuch as subjects often report that they were aware that previously studied items were included in the priming task (Bowers \& Schacter, 1990; Richardson-Klavehn, Lee, Joubran, \& Bjork, 1994). However, even though subjects may become aware that previously studied items are occurring in the priming task, they do not necessarily attempt to retrieve these items intentionally (Richardson-Klavehn et al., 1994). Indeed, it appears that when subjects are informed that items will be repeated, as the subjects were in all of the experiments of the present study, they are least likely intentionally to try to retrieve previously studied items - even in wordstem completion tasks (Bowers \& Schacter, 1990).

Furthermore, it appears that latency measures of priming are even less likely to be influenced by such strategies (Mitchell, 1993). For instance, Brown, Neblett, Jones, and Mitchell (1991) created conditions in a picture-naming experiment that would seem to be optimal for the use of explicit retrieval strategies, and yet no effect on priming was observed. They found that blocking old and new items and informing subjects about the old/new status of the blocked items did not affect the magnitude of nam- 
ing latency priming in comparison with conditions under which old and new items were randomly mixed. In summary, although it is impossible to rule out completely the possibility that the subjects used explicit memory strategies, it seems unlikely that such strategies significantly affected performance in the present study. Furthermore, there is no reason to suspect that explicit retrieval of previously encountered items was more likely in the fade-in condition than in the instant condition; the use of such strategies cannot, therefore, account for the different patterns of results obtained in the two conditions.

It should be noted that, according to the view advocated here, subjects do engage in retrieval in word-naming tasks and in other information-processing tasks used to measure priming. Subjects retrieve any relevant information that will allow them to carry out the task, and some of this relevant information may be episodic information available in memory from a prior study episode. Therefore, in some sense, priming effects may be regarded as a "contamination" of task performance by episodic information from the study episode.

\section{The Information Availability Model}

It is argued that the present results can best be explained in terms of the information available to the subject for accomplishing the task of naming visually presented words. For relatively familiar items presented clearly, the amount of available perceptual information, combined with information from prior encounters with the items, is likely to be large relative to the information available from any one specific encounter (study episode). Consistent with this view is the fact that subjects can easily carry out the task without having been exposed to a prior study episode (baseline performance level is high). Under such circumstances, the relatively small amount of information available from a specific study episode will have only a small effect on overall performance. Furthermore, not only will priming effects be small, but, as demonstrated earlier, variability in the amount of information available from a study episode may not be reflected in variability of the obtained priming effects. When the amount of nonstudy information available was reduced in the present experiments, constraints on priming effects were reduced, resulting in larger priming effects and greater sensitivity of priming to experimental manipulations.

If baseline performance level is regarded as a measure of task difficulty, then manipulations that make a task more difficult will also increase the magnitude and the sensitivity to experimental variables of measured priming effects. Within the present framework, there are three ways to change task difficulty. Using less familiar, rather than more familiar, items (reducing the amount of $O$ available) will increase task difficulty. Manipulating the amount of information directly available from the presented stimuli $(P)$ will similarly affect task difficulty. Finally, the task itself can be changed such that more or less information $(T)$ is required to carry out the task successfully. All of the experiments in the present study involved a manipulation of the visual information available from the presented stimuli. Additionally, in Experiments 1 and 2, the familiarity of items (as indexed by word frequency) was manipulated. In all cases, increasing task difficulty, whether by slowing down fade-in times or by reducing familiarity with the items, resulted in an increase in the magnitude and sensitivity of priming effects. The effects of word frequency on priming were larger in the slow than in the fast fade-in conditions, and the effect of the fade-in manipulation was larger for lowfrequency than for high-frequency words. Furthermore, the effects on priming of additional repetitions of the study items and of the length of delay between the study episode and the priming test were larger when the items faded in gradually than when they were presented instantaneously. Thus, reducing the information that was available to the subject for carrying out the word-naming task resulted in slower baseline naming performance, larger measured priming effects, and increased sensitivity of priming effects to experimental manipulations.

The information availability model shows how the magnitude of measured priming effects may not be directly related to the amount or the strength of information that is available from a prior study episode. In other words, a given information-processing task may provide only a limited window on the underlying priming variability, and if the variability of priming resulting from an experimental manipulation falls outside this window, then the effect of the manipulation cannot be detected. The information requirements of a given retrieval (informationprocessing) task, together with the availability of relevant information from any source, determines the extent to which a prior study episode will affect performance. In this context, it is important to consider exactly what is relevant information for a given retrieval task. The information available from all prior similar encounters with an item is probably relevant to the subject's decision in an identification task (Whittlesea, 1987; Whittlesea \& Brooks, 1988). That is not the case in explicit-memory tasks. In explicit-memory tasks, the crucial decision is not about the identity of an item but rather about whether the item was encountered during a specific study episode. The information that is relevant to the decision to generate an item in free recall, or to the selection of an old or a new response in a recognition-memory task, is the information that is available from the prior study episode. In that sense, performance in explicit-memory tasks is directly related to the amount or the strength of information that is available from the prior study episode. Although many information-processing components may be involved in explicit-memory tasks (e.g., all the processes required for item identification are required in recognition-memory tasks), the critical decision in such tasks is heavily dependent on the information from the study episode. Explicit-memory tasks cannot be carried 
out in the absence of a study episode, whereas performance on priming tasks generally is very good even in the absence of a study episode. Therefore, although, according to the present view, priming effects and recognitionmemory performance are both mediated by episodic information from the study episode, it may not be possible to observe a direct relationship between explicit-memory performance and priming in many circumstances. However, according to the present hypothesis, conditions that increase the influence on priming effects of the information from the study episode should also increase the likelihood of observing a direct relationship between explicit-memory performance and the magnitude of priming effects. This is exactly what was observed in Experiment 2 . When the availability of perceptual information was drastically reduced and the magnitude of priming effects greatly increased, a direct relationship between priming and recognition-memory performance was detectable. A similar relationship between priming and recognition memory was reported by Jernigan and Ostergaard (1993) in a mixed group of normal subjects and patients with varying degrees of memory impairments. In this case, the relationship could be observed only after the variability in the priming measure associated with baseline performance level had been removed in a multiple regression analysis.

\section{Modality Effects}

In the comparison of priming and explicit-memory tasks, it seems important to consider the types of information required to carry out the tasks and the availability of this information from any source. Such considerations may also be important in the evaluation of the impact on priming of manipulations of the study task. For instance, a change in modality from study to test often severely reduces, indeed sometimes completely removes, priming effects, whereas such a change has little or no effect on explicit-memory measures (see, e.g., Kirsner, Milech, \& Standen, 1983). It could be argued that these modality effects on priming are in conflict with the present argument that priming variability is often too constrained for the effects of other variables to be observed. However, it is not argued here that even very large effects of manipulations are not detectable with standard priming measures. Rather, it is suggested that information-processing tasks provide only a limited window on the underlying priming variability. Therefore, a very large effect could be detectable, whereas a moderate effect might not be. The effects of modality change on priming are often very large. For instance, in tachistoscopic identification tasks, it is often the case that no significant priming effects are observed in the crossmodal conditions (Clarke \& Morton, 1983; Jacoby \& Dallas, 1981; Rajaram \& Roediger, 1993; Richardson-Klavehn et al., 1994). It is acknowledged that, in many perceptual priming experiments, priming effects have been observed in crossmodal conditions (for reviews, see Richardson-
Klavehn \& Bjork, 1988; Roediger \& McDermott, 1993). However, these effects are small and often nonsignificant, which testifies to the fact that a change in modality from study to test has a very large effect on priming measures. Thus, that modality-change effects on priming may be detectable, although the effects of other manipulations may not be, is not inconsistent with the present view.

It is worth noting that the modality-change dissociation between explicit memory and priming measures is not entirely consistent across studies. Small but significant effects of modality change have been reported in recognition-memory tasks (Kirsner, 1974). In particular, Jacoby and Dallas (1981) reported significant effects of modality change on both perceptual-identification priming and recognition-memory measures that were obtained within the same experiments. Relatively large and consistent effects of modality change have been reported in explicit word-stem or word-fragment cued recall, and these effects are often as large as those obtained in implicit stem or fragment completion (Challis \& Sidhu, 1993; Craik, Moscovitch, \& McDowd, 1994). Furthermore, modality effects are not observed in all priming tasks. In tasks often referred to as conceptual priming tasks, such as answering general knowledge questions or generating exemplars from a specified semantic category, priming is not affected by modality change (Challis \& Sidhu, 1993; Srinivas \& Roediger, 1990). In other words, modality effects are not easily understood in terms of differences between explicit memory and priming processes or systems. Modality effects are probably better understood in terms of the relationship between the processing operations employed during the acquisition of information and those required at retrieval. This has been referred to as transfer-appropriate processing (Morris, Bransford, \& Franks, 1977; Roediger et al., 1989) or as compatibility of processing operations (Hintzman, 1990).

Within the present framework, priming effects are determined by the relationship between the amount of relevant information available in memory from the study episode and the amount of relevant information available from other sources. It does not seem surprising that a study task that involves the visual processing of words produces more information in memory that is relevant to a subsequent visual word-identification task than does an acquisition task that involves auditory processing. In recognition-memory tasks, the cue (test item) is always identified, and the decision as to whether the test item occurred during the study episode is made subsequent to identification. It seems plausible that any information that is available from the study episode is relevant to the recognition-memory decision, and therefore that the modality of the test item is of less importance. Explicitmemory tasks, such as word-stem or word-fragment cued recall, do require modality-specific perceptual processing. On each trial, the subject has to retrieve an item from the study episode that is consistent with a partial visual cue. Thus, visual processing at study would benefit this 
retrieval process more than would auditory processing, and modality effects are to be expected in such explicitmemory tasks.

\section{Conclusion}

In light of the present results and of previous reports of conflicting effects of experimental variables on priming, it seems to be necessary to consider priming effects in relation to the information-processing characteristics of a given priming task. The memory component of a priming task is often relatively small compared to other information-processing components of the task, and these other information-processing components are likely to modulate and constrain the memory effect (Hintzman \& Hartry, 1990; Ostergaard, 1992, 1995; Ostergaard \& Jernigan, 1993). The present information availability approach focuses on how information that is available from different sources interacts to determine performance in the retrieval task. In common with the views expressed by others (e.g., Jacoby, 1983a, 1983b; Jacoby et al., 1989; Whittlesea, 1987; Whittlesea \& Brooks, 1988), priming depends on retrieval, perhaps incidental retrieval, of episodic information in memory from a specific previous encounter with an item. The importance of the characteristics of retrieval tasks has also been stressed previously. For instance, Ratcliff and McKoon (1995) and Ratcliff, McKoon, and Verwoerd (1989) demonstrated how retrieval manipulations can affect the priming effects obtained in a variety of tasks, and Ostergaard (1994) illustrated how different retrieval cues could produce different patterns of priming effects in word-identification tasks.

Such considerations, together with the findings from the present study, underscore the importance of identifying the component processes of the informationprocessing tasks employed to measure priming (Masson, 1989; Moscovitch, 1992; Tenpenny \& Shoben, 1992). The present investigation has focused on identifying the consequences of constraints on priming effects in a single word-naming task. Different constraints may affect other implicit-memory tasks, and careful analyses of such tasks may elucidate the nature of such constraints. For instance, in word-stem completion tasks, baseline performance level is not a measure of how well the subject can perform the task or of task difficulty, but is simply a measure of how likely the subject is to produce the particular solutions chosen by the experimenter. In such tasks, constraints may be related to how good a cue a particular stem is for a particular solution. The consequences of baseline variation and possible ceiling effects for priming are currently being actively debated with respect to tachistoscopic word identification. In that case, the critical issue is whether baseline performance variation and ceiling effects can account for conflicting findings obtained with memory-impaired subjects (see Hamann et al., 1995; Jernigan \& Ostergaard, 1993; Ostergaard, 1994; Ostergaard \& Jernigan, 1993, 1995). Detailed analyses of each individual information-processing task may be required in order to determine how information from a specific study episode interacts with information available from other sources to produce priming effects.

\section{REFERENCES}

BECKER, C. A., \& KiLlion, T. H. (1977). Interaction of visual and cognitive effects in word recognition. Journal of Experimental Psychology: Human Perception \& Performance, 3, 389-401.

Besner, D., \& Swan, M. (1982). Models of lexical access in visual word recognition. Quarterly Journal of Experimental Psychology, 34A, 313-325.

Bowers, J. S., \& SChacter, D. L. (1990). Implicit memory and test awareness. Journal of Experimental Psychology: Learning, Memory, \& Cognition, 16, 404-416.

Brown, A., Neblett, D., Jones, T., \& Mitchell, D. (1991). Transfer of processing in repetition priming: Some inappropriate findings. Journal of Experimental Psychology: Learning, Memory, \& Cognition, 17, 514-525

Challis, B. H., \& SidHu, R. (1993). Dissociative effect of massed repetition on implicit and explicit measures of memory. Journal of Experimental Psychology: Learning, Memory, \& Cognition, 19, 115-127.

Clark, H. H. (1973). The language-as-a-fixed-effect fallacy: A critique of language statistics in psychological research. Journal of Verbal Learning \& Verbal Behavior, 12, 335-359.

ClARKe, R., \& MORTON, J. (1983). Cross modality facilitation in tachistoscopic word recognition. Quarterly Journal of Experimental Psychology: Human Experimental Psychology, 35A, 79-96.

Craik, F. I. M., Moscovitch, M., \& McDowd, J. M. (1994). Contributions of surface and conceptual information to performance on implicit and explicit memory tasks. Journal of Experimental Psychology: Learning, Memory, \& Cognition, 20, 864-875.

DunN, J. C., \& Kirsner, K. (1988). Discovering functionally independent mental processes: The principle of reversed association. Psychological Review, 95, 91-101.

EICH, E. (1984). Memory for unattended events: Remembering with and without awareness. Memory \& Cognition, 12, 105-111.

Feustel, T. C., Shiffrin, R. M., \& Sal asoo, M. A. (1983). Episodic and lexical contributions to repetition effects in word identification. Journal of Experimental Psychology: General, 112, 309-346.

Glanzer, M., \& AdAMS, J. K. (1985). The mirror effect in recognition memory. Memory \& Cognition, 13, 8-20.

GRAF, P., \& MANDLER, G. (1984). Activation makes words more accessible, but not necessarily more retrievable. Journal of Verbal Learning \& Verbal Behavior, 23, 553-568.

Graf, P., Squire, L. R., \& Mandler, G. (1984). The information amnesic patients do not forget. Journal of Experimental Psychology: Learning, Memory, \& Cognition, 10, 164-178.

GreEne, R. L. (1990). Spacing effects on implicit memory tests. Journal of Experimental Psychology: Learning, Memory, \& Cognition, 16, 1004-1011

GREGG, V. (1976). Word frequency, recognition and recall. In J. Brown (Ed.), Recall and recognition (pp. 183-216). New York: Wiley.

HALL, J. F. (1954). Learning as a function of word frequency. American Journal of Psychology, 67, 138-140.

Hamann, S. B., Squire, L. R., \& Schacter, D. L. (1995). Perceptual thresholds and priming in amnesia. Neuropsychology, 9, 3-15.

Hayman, G. C. A., \& Tulving, E. (1989). Is priming in fragment completion based on a "traceless" memory system? Journal of Experimental Psychology: Learning, Memory, \& Cognition, 15, 941-956.

Hintzman, D. L. (1990). Human learning and memory: Connections and dissociations. Annual Review of Psychology, 41, 109-139.

Hintzman, D. L. (1993). On variability, Simpson's paradox, and the relation between recognition and recall: Reply to Tulving and Flexser. Psychological Review, 100, 143-148; discussion 149-153.

Hintzman, D. L., \& HarTRY, A. L. (1990). Item effects in recognition and fragment completion: Contingency relations vary for different subsets of words. Journal of Experimental Psychology: Learning. Memory, \& Cognition, 16, 955-969. 
JACOBY, L. L. (1983a). Perceptual enhancement: Persistent effects of an experience. Journal of Experimental Psychology: Learning, Memory, \& Cognition, 9, 21-38.

JACOBY, L. L. (1983b). Remembering the data: Analyzing interactive processes in reading. Journal of Verbal Learning \& Verbal Behavior, 22, 485-508.

JACOBY, L. L., BAKER, J. G., \& BRoOKs, L. R. (1989). Episodic effects on picture identification: Implications for theories of concept learning and theories of memory. Journal of Experimental Psychology: Learning, Memory, \& Cognition, 15, 275-281.

JACOBY, L. L., \& DALLAS, M. (1981). On the relationship between autobiographical memory and perceptual learning. Journal of Experimental Psychology: General, 110, 306-340.

Jernigan, T. L., \& OstergaARD, A. L. (1993). Word priming and recognition memory both affected by mesial temporal lobe damage. Neuropsychology, 7, 14-26.

Johnston, W. A., Dark, V. J., \& JACoBy, L. L. (1985). Perceptual fluency and recognition judgements. Journal of Experimental Psychology: Learning, Memory, \& Cognition, 11, 3-11.

KIRSNER, K. (1974). Modality differences in recognition memory for words and their attributes. Journal of Experimental Psychology, 102, 579-584

KIRSNER, K., Milech, D., \& Standen, P. (1983). Common and modalityspecific processes in the mental lexicon. Memory \& Cognition, 11, $621-630$.

Kirsner, K., Milech, D., \& Stumpfel, V. (1986). Word and picture identification: Is representational parsimony possible? Memory \& Cognition, 14, 398-408.

KuČERA, H., \& FRANCIS, W. (1967). Computational analysis of presentday American English. Providence, RI: Brown University Press.

Light, L. L., SINGH, A., \& CAPPS, J. L. (1986). Dissociation of memory and awareness in young and older adults. Journal of Clinical \& Experimental Neuropsychology, 8, 62-74.

LofTUS, G. R., \& MAsson, M. E. J. (1994). Using confidence intervals in within-subject designs. Psychonomic Bulletin \& Review, 1, 476-490.

MACLEOD, C. M. (1989). Word context during initial exposure influences degree of priming in word fragment completion. Journal of Experimental Psychology: Learning, Memory, \& Cognition, 15, 398-406.

MASSON, M. E. J. (1989). Fluent reprocessing as an implicit expression of memory for experience. In S. Lewandowsky, J. C. Dunn, \& K. Kirsner (Eds.), Implicit memory: Theoretical issues (pp. 123-138). Hillsdale, NJ: Erlbaum.

MrTCHELl, D. (1993). Implicit and explicit memory for pictures: Multiple views across the lifespan. In P. Graf \& M. Masson (Eds.), Implicit memory: New directions in cognition, development and neuropsychology (pp. 171-190). Hillsdale, NJ: Erlbaum.

MORRIS, C., BRANSFORD, J., \& FRANKS, J. (1977). Levels of processing versus transfer appropriate processing. Journal of Verbal Learning \& Verbal Behavior, 16, 519-533.

Moscovitch, M. (1992). Memory and working-with-memory: A component process model based on modules and central systems. Special issue: Memory systems. Journal of Cognitive Neuroscience, 4, 257-267.

NEELY, J. H. (1989). Experimental dissociations and the episodic/ semantic memory distinction. In H. L. Roediger III \& F. I. M. Craik (Eds.), Varieties of memory and consciousness: Essays in honour of Endel Tulving (pp. 229-270). Hillsdale, NJ: Erlbaum

Nelson, D. L., MCEvoy, C. L., \& Bajo, M. (1988). Lexical and semantic search in cued recall, fragment completion, perceptual identification, and recognition. American Journal of Psychology, 101, 465-480

Norris, D. (1984). The effect of frequency, repetition and stimulus quality in visual word recognition. Quarterly Joumal of Experimental Psychology, 36A, 507-518.

OLTON, D. (1989). Inferring psychological dissociations from experimental dissociations: The temporal context of episodic memory. In H. L. Roediger III \& F. I. M. Craik (Eds.), Varieties of memory and consciousness: Essays in honour of Endel Tulving (pp. 161-177). Hillsdale, NJ: Erlbaum.

OstergaARD, A. L. (1992). A method for judging measures of stochas- tic dependence: Further comments on the current controversy. Journal of Experimental Psychology: Learning, Memory, \& Cognition, 18, 413-420.

OSTERGAARD, A. L. (1994). Dissociations between word priming effects in normal subjects and patients with memory disorders: Multiple memory systems or retrieval? Quarterly Journal of Experimental Psychology, 47A, 331-364.

OSTERGAARD, A. L. (1995). Who is mistaken about priming in "recognition/identification" experiments? European Journal of Cognitive Psychology, 7, 1-11.

OSTERGAaRd, A. L., \& JeRnigan, T. L. (1993). Are word priming and explicit memory mediated by different brain structures? In P. Graf \& M. Masson (Eds.), Implicit memory: New directions in cognition, development and neuropsychology (pp. 327-349). Hillsdale, NJ: Erlbaum.

Ostergaard, A. L., \& Jernigan, T. L. (1995). Priming and baseline perceptual identification performance in amnesia: A reply to Hamann, Squire, and Schacter. Neuropsychology, 10, 125-130.

Parkin, A. J., ReID, T. K., \& Russo, R. (1990). On the differential nature of implicit and explicit memory. Memory \& Cognition, 18, 507-514.

Perrucher, P. (1989). The effect of spaced practice on explicit and implicit memory. British Journal of Psychology, 80, 113-130.

Perruchet, P., \& Baveux, P. (1989). Correlational analyses of explicit and implicit memory performance. Memory \& Cognition, 17, 77-86.

RAJARAM, S., \& ROEDIGER, H. L., III (1993). Direct comparison of four implicit memory tests. Journal of Experimental Psychology: Learning. Memory, \& Cognition, 19, 765-776.

RATCLIFF, R., \& McKoon, G. (1995). Bias in the priming of object decisions. Journal of Experimental Psychology: Learning, Memory, \& Cognition, 21, 754-767

Ratcliff, R., McKoon, G., \& VerWoerd, M. (1989). A bias interpretation of facilitation in perceptual identification. Journal of Experimental Psychology: Learning, Memory, \& Cognition, 15, 378-387.

RichaRDSON-KLAVEHN, A., \& BJORK, R. A. (1988). Measures of memory. Annual Review of Psychology, 39, 475-543.

Richardson-Klavehn, A., Lee, M. G., Joubran, R., \& Bjork, R. A. (1994). Intention and awareness in perceptual identification priming. Memory \& Cognition, 22, 293-312.

RoEDiger, H. L., III, \& Challis, B. H. (1992). Effects of exact repetition and conceptual repetition on free recall and primed word-fragment completion. Journal of Experimental Psychology: Learning, Memory. \& Cognition, 18, 3-14.

Roediger, H. L., III, \& MCDERmott, K. B. (1993). Implicit memory in normal human subjects. In F. Boller \& J. Grafman (Eds.), Handbook of neuropsychology (Vol. 8, pp. 63-131). Amsterdam: Elsevier.

Roediger, H. L., III, Weldon, M. S., \& Challis, B. H. (1989). Explaining dissociations between implicit and explicit measures of retention: A processing account. In H. L. Roediger III \& F. I. M. Craik (Eds.), Varieties of memory and consciousness: Essays in honour of Endel Tulving (pp. 3-41). Hillsdale, NI: Erlbaum.

RoEdiger, H. R., III, Weldon, M. S., STADleR, M. L., \& RiegleR, G. L. (1992). Direct comparison of two implicit memory tests: Word fragment and word stem completion. Journal of Experimental Psychology: Learning, Memory, \& Cognition, 18, 1251-1269.

Scarborough, D. L., Cortese, C., \& Scarborough, H. S. (1977) Frequency and repetition effects in lexical memory. Journal of Ex perimental Psychology: Human Perception \& Performance, 3, 1-17.

Scarborough, D. L., Gerard, L., \& Cortese, C. (1979). Accessing lexical memory: The transfer of word repetition effects across task and modality. Memory \& Cognition, 7, 3-12.

Shimamura, A. P. (1985). Problems with the finding of stochastic independence as evidence for multiple memory systems. Bulletin of the Psychonomic Society, 23, 506-508.

SnOdGrass, J. G., \& FEenan, K. (1990). Priming effects in picture fragment completion: Support for the perceptual closure hypothesis Journal of Experimental Psychology: General, 119, 276-296.

SQuiRE, L. R. (1992). Memory and the hippocampus: A synthesis from findings with rats, monkeys and humans. Psychological Review, 99 , 195-231.

SQuire, L. R., Shimamura, A. R., \& Graf, P. (1987). Strength and du- 
ration of priming effects in normal subjects and amnesic patients Neuropsychologia, 25, 195-210.

SRINIVAS, K., \& ROEDIGER, H. L., III (1990). Classifying implicit memory tests: Category association and anagram solution. Journal of Memory \& Language, 29, 389-412.

StanNers, R. F., JastrzembSki, J. E., \& Westbrook, A. (1975). Frequency and visual quality in a word-non-word classification task. Journal of Verbal Learning \& Verbal Behavior, 14, 259-264.

TenPenny, P. L., \& Shoben, E. J. (1992). Component processes and the utility of the conceptually-driven/data-driven distinction. Journal of Experimental Psychology: Learning, Memory, \& Cognition, 18, 25-42.

Tulving, E., \& SCHACTER, D. L. (1990). Priming and human memory systems. Science, 247, 301-306.

Tulving, E., Schacter, D. L., \& Stark, H. A. (1982). Priming effects in word-fragment completion are independent of recognition memory.
Journal of Experimental Psychology: Learning, Memory, \& Cognition, 8, 336-342.

Warrington, E. K., \& WeiskrantZ, L. (1968). New method of testing long-term retention with special reference to amnesic patients. $\mathrm{Na}$ ture, 217, 972-974.

Whit TLESEA, B. W. [A.] (1987). Preservation of specific experiences in the representation of general knowledge. Journal of Experimental Psychology: Learning, Memory, \& Cognition, 13, 3-17.

WhitTlesea, B. W. A., \& Brooks, L. R. (1988). Critical influence of particular experiences in the perception of letters, words, and phrases. Memory \& Cognition, 16, 387-399.

(Manuscript received March 7, 1995;

revision accepted for publication December 9, 1996.) 